\title{
The impact of vorticity waves on the shock dynamics in core-collapse supernovae
}

\author{
César Huete, ${ }^{1 \star}$ Ernazar Abdikamalov ${ }^{2}$ and David Radice ${ }^{3,4}$ \\ ${ }^{1}$ Fluid Mechanics Group, Escuela Politécnica Superior, Universidad Carlos III de Madrid, E-28049 Madrid, Spain \\ ${ }^{2}$ Department of Physics, School of Science and Technology, Nazarbayev University, Astana 010000, Kazakhstan \\ ${ }^{3}$ Institute for Advanced Study, 1 Einstein Drive, Princeton, NJ 08540, USA \\ ${ }^{4}$ Department of Astrophysical Sciences, Princeton University, 4 Ivy Lane, Princeton, NJ 08544, USA
}

Accepted 2017 December 27. Received 2017 December 21; in original form 2017 September 21

\begin{abstract}
Convective perturbations arising from nuclear shell burning can play an important role in propelling neutrino-driven core-collapse supernova explosions. In this work, we analyse the impact of vorticity waves on the shock dynamics, and subsequently on the post-shock flow, using the solution of the linear hydrodynamics equations. As a result of the interaction with the shock wave, vorticity waves increase their kinetic energy, and a new set of entropic and acoustic waves is deposited in the post-shock region. These perturbations interact with the neutrino-driven turbulent convection that develops in that region. Although both vorticity and acoustic waves inject non-radial motion into the gain region, the contribution of the acoustic waves is found to be negligibly small in comparison to that of the vorticity waves. On the other hand, entropy waves become buoyant and trigger more convection. Using the concept of critical neutrino luminosity, we assess the impact of these modes on the explosion conditions. While the direct injection of non-radial motion reduces the critical neutrino luminosity by $\sim 12$ per cent for typical problem parameters, the buoyancy-driven convection triggered by entropy waves reduces the critical luminosity by $\sim 17-24$ per cent, which approximately agrees with the results of three-dimensional neutrino-hydrodynamics simulations. Finally, we discuss the limits of validity of the assumptions employed.
\end{abstract}

Key words: shock waves -turbulence - supernovae: general.

\section{INTRODUCTION}

First described by Baade \& Zwicky (1934) as the 'transition of an ordinary star into a neutron star', core-collapse supernovae (CCSNe) are the powerful explosions of massive stars that occur at the end of their lives (e.g. Bethe 1990). Upon reaching its maximum mass, the iron core becomes unstable, initiating a collapse to a protoneutron star (PNS). The shock wave launched at core bounce quickly loses its energy and stalls at a radius of $\sim 150 \mathrm{~km}$. In order to produce an explosion and leave behind a stable neutron star, the shock must recover within a few hundreds of milliseconds and expel the stellar envelope (e.g. O'Connor \& Ott 2011; Ott et al. 2011; Ugliano et al. 2012). Otherwise, a black hole (BH) forms (e.g. Nadezhin 1980; Lovegrove \& Woosley 2013; Kashiyama \& Quataert 2015; Adams et al. 2017). The details of how this occurs remain unclear, constituting one of the longest standing open questions in modern astrophysics (see e.g. Janka et al. 2012; Burrows 2013; Foglizzo et al. 2015; Müller 2016; Radice et al. 2017b, for recent reviews).

^E-mail: chuete@ing.uc3m.es
A key ingredient for producing the explosion is the neutrino emission by the newly born PNS, which deposits energy behind the shock and establishes a negative entropy gradient that drives vigorous neutrino-driven convection. Together with the standingaccretion shock instability (SASI), these multidimensional hydrodynamic effects create favourable conditions for shock revival (e.g. Burrows, Hayes \& Fryxell 1995; Herant 1995; Janka \& Mueller 1996; Blondin, Mezzacappa \& DeMarino 2003; Foglizzo, Scheck \& Janka 2006; Yamasaki \& Yamada 2006; Hanke et al. 2012, 2013; Dolence et al. 2013; Murphy, Dolence \& Burrows 2013; Ott et al. 2013; Fernández et al. 2014; Takiwaki, Kotake \& Suwa 2014; Abdikamalov et al. 2015; Cardall \& Budiardja 2015; Fernandez 2015; Lentz et al. 2015; Melson, Janka \& Marek 2015; Radice, Couch \& Ott 2015; Bruenn et al. 2016; Radice et al. 2016; Roberts et al. 2016). If present, rapid rotation may facilitate explosion via the magnetorotational mechanism (Burrows et al. 2007; Mösta et al. 2014, 2015, see also Takiwaki, Kotake \& Suwa 2016; Summa et al. 2018).

Couch \& Ott (2013) demonstrated that the perturbations arising from the turbulent convection in $\mathrm{Si}$ and $\mathrm{O}$ burning shells in CCSN progenitors may help to revive the shock. As the iron core collapses, 
the perturbations follow the core and accrete towards the centre. Because of the converging geometry of the flow and conservation of angular momentum, the perturbations amplify significantly during collapse (Kovalenko \& Eremin 1998; Lai \& Goldreich 2000; Takahashi \& Yamada 2014). Further amplification occurs at shock crossing. For example, Abdikamalov et al. (2016) find that, due to interaction with the shock, the kinetic energy of vorticity waves increases by a factor of $\sim 2$. Once in the post-shock region, the fluctuations contribute to the non-radial flow in the gain region, creating a more favourable condition for producing explosion (Couch \& Ott 2015; Couch et al. 2015; Müller \& Janka 2015; Abdikamalov et al. 2016; Burrows et al. 2016; Takahashi et al. 2016; Radice et al. 2017a).

Müller et al. (2016) presented three-dimensional (3D) simulation of the last minutes of $\mathrm{O}$ shell burning in an $18 \mathrm{M}_{\odot}$ progenitor star. Prior to collapse, they observed vigorous convection with Mach number of $\sim 0.1$ and dominant angular wavenumber of $l=2$. Full 3D neutrino-hydrodynamics simulation of this model yielded strong explosion after the accretion of the $\mathrm{O}$ shell through the shock, whereas in a model with artificially suppressed pre-collapse convection, no explosion was observed (Müller et al. 2017). The reduction of the critical (i.e. minimum) neutrino luminosity for producing explosion due to these perturbations was estimated to be $\sim 20$ per cent, which is roughly in agreement with the analytic predictions of Müller et al. (2016). Recently, Collins, Müller \& Heger (2018) investigated the properties of $\mathrm{Si}$ and $\mathrm{O}$ shell burning in a broad range of presupernova models with zero-age main sequence (ZAMS) masses between 9.45 and $35 \mathrm{M}_{\odot}$. They found that the progenitor models between 16 and $26 \mathrm{M}_{\odot}$ exhibit large-scale convective motions with high Mach numbers in the $\mathrm{O}$ shells, which are favourable conditions for producing perturbation-aided neutrino-driven explosions (Müller \& Janka 2015). On the other hand, strong perturbations were rarely observed in the $\mathrm{Si}$ shells.

The emerging qualitative picture of how the progenitor asphericities impact the explosion condition is as follows. The convective vorticity waves distort the spherical isodensity surfaces of the progenitor star, creating Eulerian density perturbations at a given radius (Mao et al. 2015). When these density and vorticity perturbations encounter and cross the shock, they generate a post-shock field of entropy, vorticity, and acoustic perturbations. These perturbations then become buoyant and contribute to the buoyancy-driven turbulence in the post-shock region, which creates more favourable conditions for producing explosion (Müller \& Janka 2015).

In order to gain the full understanding of how these perturbations affect the explosion dynamics, it is necessary to understand the physics of shock-turbulence interaction, starting with linear order. With this premise, Abdikamalov et al. (2016) studied the effect of entropy and vorticity perturbations using a linear perturbation theory known as the linear interaction analysis (LIA) (e.g. Ribner 1953; Mahesh, Moin \& Lele 1996). These two represent two of the three components of a generic turbulent flow, the third being acoustic waves (Kovasznay 1953; Chu \& Kovasznay 1957). They found that the kinetic energy of these fluctuations increases by a factor of $\sim 2$ as they cross the shock. Assuming direct injection of this energy into the post-shock region, they estimated that these perturbations can reduce the critical neutrino luminosity for producing explosion by $\sim 12$ per cent. While this is an important finding, the physics of shock-turbulence in CCSNe, even at linear level, is not yet completely understood. As noted by Müller et al. (2017), the buoyancy plays a dominant role in generating post-shock turbulence. Moreover, the acoustic waves generated by in-falling entropy and vorticity perturbations (Kovalenko \& Eremin 1998; Foglizzo \&
Tagger 2000; Müller 2016) will affect the shock dynamics and the post-shock flow. Finally, the impact of perturbations on the nuclear dissociation rate itself should also be taken into account. These aspects are missing from the analysis of Abdikamalov et al. (2016).

In this work, it is investigated the interaction between accretion shocks and turbulent fluctuations in further detail. The study is based on the solution of the linearized hydrodynamics equations in the post-shock region, which permits to capture the full temporal evolution of shock-vorticity interaction. The mathematical formalism describing the post-shock perturbation flow is similar to that employed in theoretical works on Richtmyer-Meshkov-type flows (Wouchuk 2001a,b; Cobos-Campos \& Wouchuk 2014) and analogous to that used in canonical interactions of non-reactive and reactive shocks with turbulent flows (Wouchuk, Huete Ruiz de Lira \& Velikovich 2009; Huete et al. 2017). This improved formalism allows us to take into account the perturbation of the nuclear dissociation itself, which was not included in Abdikamalov et al. (2016). As demonstrated below, this effect is found to be important in the turbulent kinetic amplification factor, with the parametric trends and the asymptotic values being significantly affected.

This is the first in a series of two papers. This paper is dedicated to the study of the interactions of accretion shocks with vorticity waves, while the second will study the interactions with density perturbations generated due to differential in-fall. The aim of this series of works is to establish in detail the linear physics of interaction of shocks with hydrodynamic turbulence in CCSNe.

The rest of the paper is organized as follows. Section 2 presents the problem formulation and the solution method. In Section 3, an analysis of the interaction of shock waves with individual vorticity waves is presented, while Section 4 focuses on the interaction of shocks with isotropic field of vorticity waves. The base-flow properties for the shock Mach number and the dissociation degree are computed in Section 5. In Section 6, it is discussed the implication of the results on the explosion condition of CCSNe. Finally, conclusions are presented in Section 7.

\section{PROBLEM FORMULATION}

\subsection{Perturbation-free flow}

Let us consider an expanding shock wave placed at $r=R_{\text {shock }}(t)$ that separates the in-falling flow ahead of shock front $r>R_{\text {shock }}$, denoted with subscript 1 , and the downstream post-shock flow identified with subscript 2 in $r<R_{\text {shock }}$ (see Fig. 1 for clarification). In the thinshock limit, when the radius of the shock is much larger than the accretion-shock thickness $R_{\text {shock }} \gg l$, the variation of the different flow variables across the shock is readily obtained through the radial integration of the conservation equations, yielding

$\rho_{1}\left(u_{1}^{\prime}+\dot{R}_{\text {shock }}\right)=\rho_{2}\left(u_{2}^{\prime}+\dot{R}_{\text {shock }}\right)$,

$p_{1}+\rho_{1}\left(u_{1}^{\prime}+\dot{R}_{\text {shock }}\right)^{2}=p_{2}+\rho_{2}\left(u_{2}^{\prime}+\dot{R}_{\text {shock }}\right)^{2}$,

$e_{1}+\frac{p_{1}}{\rho_{1}}+\frac{1}{2}\left(u_{1}^{\prime}+\dot{R}_{\text {shock }}\right)^{2}=e_{2}+\frac{p_{2}}{\rho_{2}}+\frac{1}{2}\left(u_{2}^{\prime}+\dot{R}_{\text {shock }}\right)^{2}$

for the mass, momentum, and energy conservation equations, respectively. The symbols $u^{\prime}, \rho, p$, and $e$ refer to the bulk velocity in a reference frame placed at the centre of the star, density, pressure, and internal energy of the gas, respectively. The sign convention adopted, consistent with Fig. 1, is based on the characteristics of the flow properties in the particular physical scenario: an in-falling 


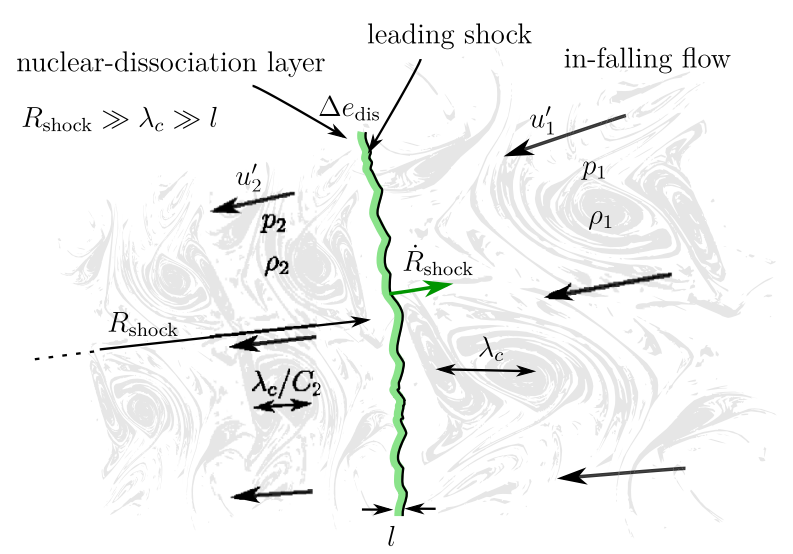

Figure 1. Scheme of the accretion shock expanding through the in-falling mass and characteristic scales: shock radius $R_{\text {shock}}$, shock thickness $l$, and characteristic perturbation wavelength $\lambda_{\mathrm{c}}$. Velocities are measured in the centre-of-the-star reference frame.

flow encountering an expanding shock. The velocities are then defined positive, $u_{1}^{\prime}>0, \dot{R}_{\text {shock }}>0$, for the star gas moving inwards and for the shock wave travelling outwards, respectively. Notice that, for non-negligible accretion shock thicknesses, the mass equation (1a) should then include the term involving the divergence of the post-shock expanding gas within the dissociation layer.

When the shocked flow, modelled as a perfect gas with the polytropic index $\gamma=4 / 3$, is affected by nuclear dissociation effects occurring in a thin layer right behind the shock, the variation of the internal energy can be computed as

$e_{1}-e_{2}=\frac{1}{\gamma-1} \frac{p_{1}}{\rho_{1}}-\frac{1}{\gamma-1} \frac{p_{2}}{\rho_{2}}+\Delta e_{\mathrm{dis}}$,

with $\gamma$ assumed constant through the interaction process, and $\Delta e_{\mathrm{dis}}$ referring to the energy per unit mass employed in dissociating the nuclei.

Following Fernández \& Thompson (2009a,b), the nuclear dissociation energy in stalled conditions can be scaled using only the free-fall speed squared, $\Delta e_{\mathrm{dis}}=\varepsilon v_{\mathrm{FF}}^{2} / 2$, with $\varepsilon$ being the characteristic dimensionless nuclear dissociation parameter. As shown below in Section $5, \varepsilon$ typically ranges from 0.2 to 0.4 in CCSN models. Assuming that gas particles in the in-falling matter are isotropically accelerated up to the shock encounter, which is equivalent to saying that the Bernoulli parameter is zero above the shock, it is found that

$\frac{1}{2} v_{\mathrm{FF}}^{2}=\frac{G \mathcal{M}}{R_{\text {shock }}}=\frac{1}{2} u_{1}^{\prime 2}+\frac{\gamma}{\gamma-1} \frac{p_{1}}{\rho_{1}}$,

where $G$ is the gravitational constant and $\mathcal{M}$ is the gravitating mass.

For non-stalled shock conditions, the dissociation energy parameter $\varepsilon$ should be rescaled to include the effect of the expanding shock speed, so that

$\Delta e_{\mathrm{dis}}=\varepsilon\left[\frac{1}{2} u_{1}^{2}+\frac{\gamma}{\gamma-1} \frac{p_{1}}{\rho_{1}}\right]$,

with $u_{1}=u_{1}^{\prime}+\dot{R}_{\text {shock }}$ being the upstream flow velocity in the shock reference frame. The shock Mach number $M_{1}=u_{1} / a_{1}$, with $a_{1}=\left(\gamma_{1} p_{1} / \rho_{1}\right)^{1 / 2}$ defining the speed of sound upstream, is conveniently introduced to rewrite nuclear dissociation energy as

$\frac{\gamma^{2}-1}{2} \frac{\Delta e_{\mathrm{dis}}}{a_{1}^{2}}=\varepsilon \frac{\gamma+1}{2}\left(1+\frac{\gamma-1}{2} M_{1}^{2}\right)$.

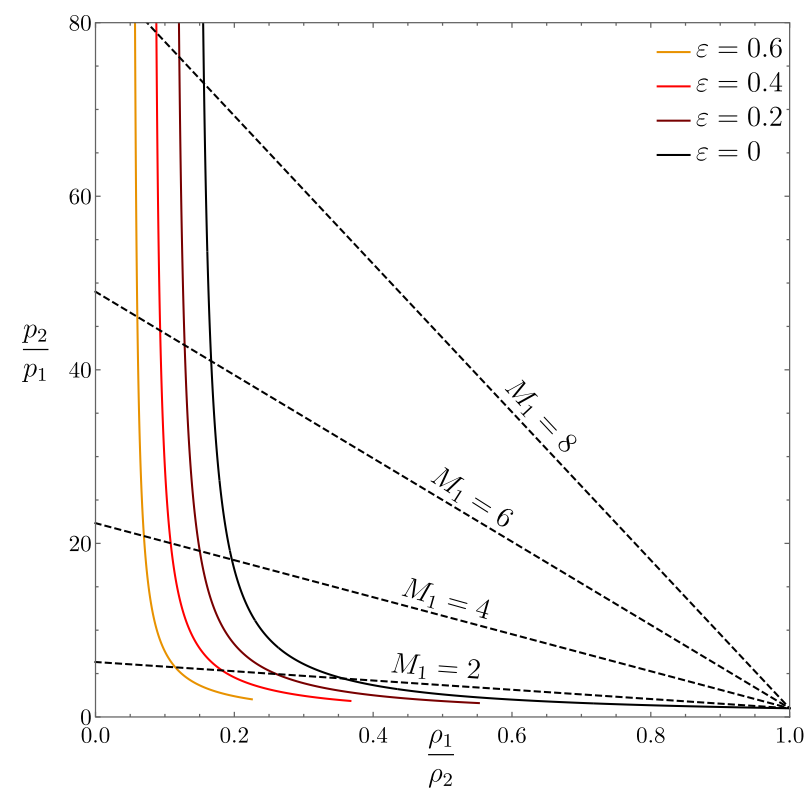

Figure 2. Hugoniot curves (solid lines) and Rayleigh lines (dashed lines) for several values of the dissociation energy parameter $\varepsilon=0,0.2,0.4$, and 0.6 .

In the stalled-shock limit the shock is mainly supported by the in-falling matter so that, $\dot{R}_{\text {shock }} / u_{1}^{\prime} \ll 1$ and then $u_{1}^{\prime} \sim u_{1}$.

Taking $\varepsilon$ and Mach as the independent parameters, the values of which may vary within the range established from numerical simulations of CCSNe (see Section 5), the fluid properties behind the shock are expressed in the form

$C_{2}=\frac{\rho_{2}}{\rho_{1}}=\frac{u_{1}}{u_{2}}=\frac{(\gamma+1) M_{1}^{2}}{(\gamma-\kappa) M_{1}^{2}+1}$

and

$P_{2}=\frac{p_{2}}{\rho_{1} u_{1}^{2}}=\frac{\gamma M_{1}^{2}(1+\kappa)+1}{\gamma(\gamma+1) M_{1}^{2}}$

for post-shock density and pressure, with $u_{2}=u_{2}^{\prime}+\dot{R}_{\text {shock }}$ being the post-shock flow speed in the shock reference frames. The Mach number of the fluid particles leaving the shock is

$M_{2}=\frac{u_{2}}{a_{2}}=\left(\gamma C_{2} P_{2}\right)^{-1 / 2}=\left[\frac{(\gamma-\kappa) M_{1}^{2}+1}{\gamma M_{1}^{2}(1+\kappa)+1}\right]^{1 / 2}$,

with the function

$\kappa=\left[\left(1-M_{1}^{-2}\right)^{2}+\varepsilon(\gamma+1)\left(\gamma-1+2 M_{1}^{-2}\right)\right]^{1 / 2}$

accounting for the dimensionless endothermic parameter $\varepsilon$. For nonreacting shock waves the value of $\kappa=1-M_{1}^{-2}$, thereby reducing equations (6)-(8) to the well-known adiabatic Rankine-Hugoniot relationships.

The effect of nuclear dissociation on the post-shock flow density and pressure is easily analysed through Fig. 2, with the final values provided by the intersection of the Rayleigh line (for a constant Mach number propagation) and the non-adiabatic RankineHugoniot curve. It is found that higher pressures and densities are required downstream to get the shock with the same Mach number, if endothermic transformations take place through the shock wave. The maximum energy that can be employed in the nuclear dissociation process is found in the extreme limit $1-\varepsilon \ll 1$, which provides limiting conditions for the post-shock gas: post-shock Mach number and temperature that tends to zero, and density that tends to infinity. 

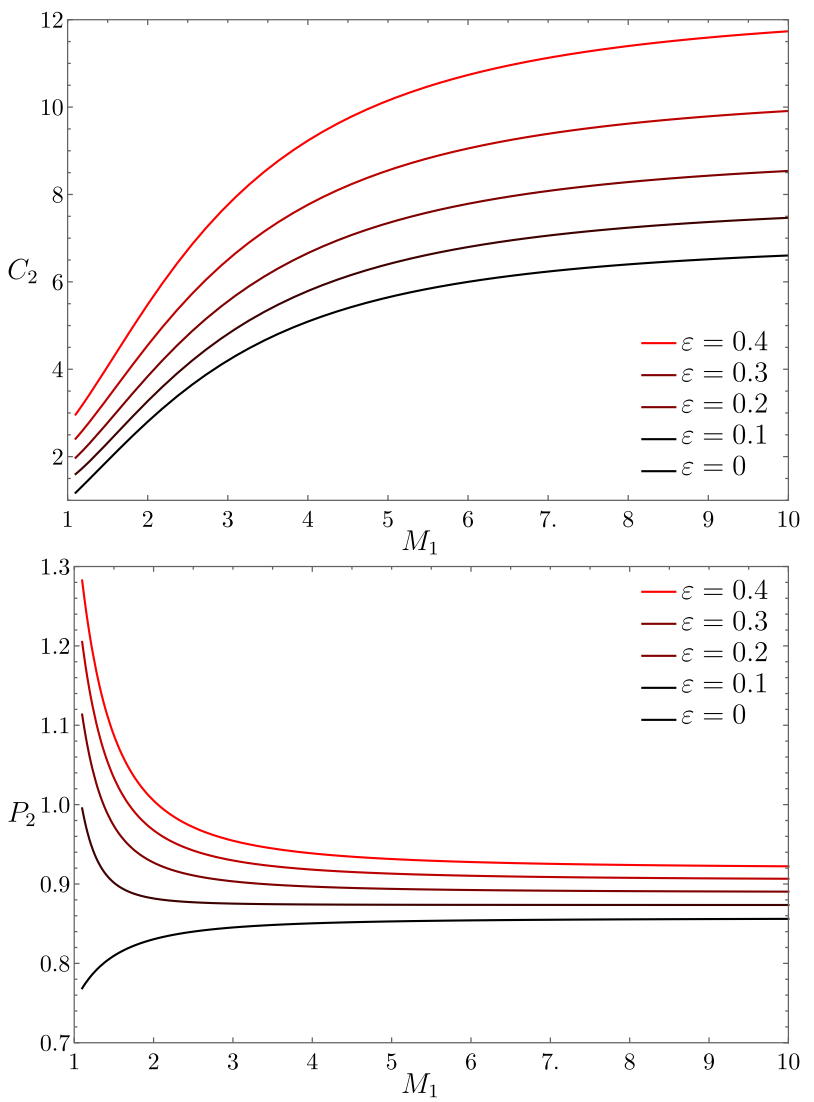

Figure 3. Mass compression ratio $C_{2}$ (top) and pressure amplification $P_{2}$ (bottom) as a function of the incident Mach number $M_{1}$ for several values of the dissociation energy parameter $\varepsilon=0,0.1,0.2,0.3$, and 0.4 .

That is, all the kinetic and thermal energy of the in-falling gas are used in dissociating the nuclei. The corresponding Hugoniot curve collapses into the vertical axis and the finite values of pressure are given by the intersection with the Rayleigh lines.

Equations (6) and (7) are computed in Fig. 3 as a function of the Mach number, $M_{1}$. Both $C_{2}$ and $P_{2}$ increase with $\varepsilon$ for the same value of $M_{1}$. Unlike regular detonations, where chemical energy release does not depend on shock intensity since the reaction is selfsustained once triggered, nuclear dissociation degree does depend on upstream Mach number. Thus, the function $\kappa$ approaches the value $\left[1+\left(\gamma^{2}-1\right) \varepsilon\right]^{1 / 2}$ in the strong-shock limit, $M_{1} \gg 1$, then yielding

$\left.C_{2}\right|_{M_{1} \gg 1}=\frac{\gamma+1}{\gamma-\left[1+\left(\gamma^{2}-1\right) \varepsilon\right]^{1 / 2}}$

and

$\left.P_{2}\right|_{M_{1} \gg 1}=\frac{1+\left[1+\left(\gamma^{2}-1\right) \varepsilon\right]^{1 / 2}}{\gamma+1}$

for the post-shock density and pressure values, in agreement with Fig. 3. The mass-compression ration $C_{2}$ is found to diverge, and $P_{2}$ approaches unity in the double limit $M_{1} \gg 1,1-\varepsilon \ll 1$.

\subsection{Perturbation problem}

The shock position and the post-shock flow variables are affected by the non-uniformities developed by the in-falling matter along its way to the shock encounter. Assumed sufficiently weak, the

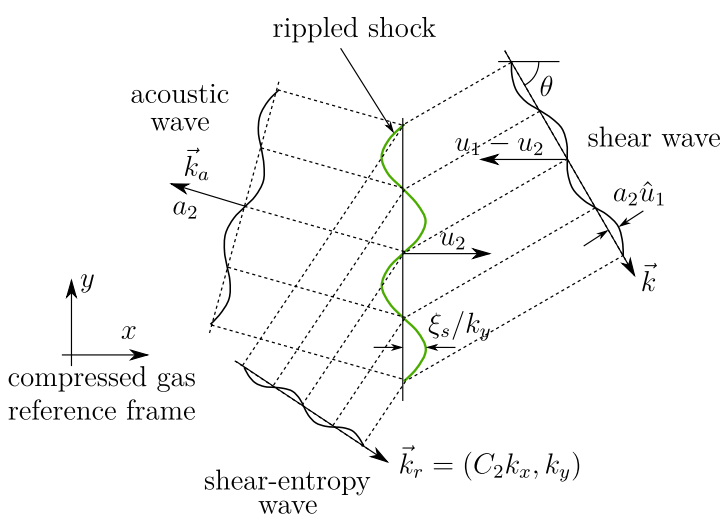

Figure 4. Scheme of the shock-shear-wave interaction in the compressed gas reference frame.

upstream and the downstream linear disturbances can be characterized in terms of acoustic, entropy, and vortical modes. As this work focuses on the effect of turbulent flows made of shear waves exclusively, the interaction with monofrequency vorticity perturbations is first described to characterize the shock and post-shock flow dynamics as a function of upstream relative vortex stretching.

The upstream shear wave in the in-falling gas reference frame $\left(x_{1}, y_{1}\right)$ is determined by the divergence-free velocity perturbation wave, namely

$\bar{u}_{1}\left(x_{1}, y_{1}\right)=\frac{u_{1}-\left\langle u_{1}\right\rangle}{\left\langle a_{2}\right\rangle}=\hat{u}_{1} \cos \left(k_{x} x_{1}\right) \cos \left(k_{y} y_{1}\right)$,
$\bar{v}_{1}\left(x_{1}, y_{1}\right)=\frac{v_{1}-\left\langle v_{1}\right\rangle}{\left\langle a_{2}\right\rangle}=\hat{u}_{1} \frac{k_{x}}{k_{y}} \sin \left(k_{x} x_{1}\right) \sin \left(k_{y} y_{1}\right)$

for the streamwise and crosswise perturbations, respectively. The brackets denote the time-averaged mean value of the flow variable, which is effectively null for the upstream velocity in the stagnant gas reference frame. The dimensionless factor $\hat{u}_{1}$ stands for the amplitude of the upstream velocity disturbances and $\boldsymbol{k}=\left(k_{x}, k_{y}\right)$ is the upstream wavenumber vector. The associated non-dimensional vorticity wave, $\bar{\omega}_{1}\left(x_{1}, y_{1}\right)=\partial \bar{v}_{1} /\left(\partial k_{y} x_{1}\right)-\partial \bar{u}_{1} /\left(\partial k_{y} y_{1}\right)$, is

$\bar{\omega}_{1}\left(x_{1}, y_{1}\right)=\hat{u}_{1}\left(1+\frac{k_{x}^{2}}{k_{y}^{2}}\right) \cos \left(k_{x} x_{1}\right) \sin \left(k_{y} y_{1}\right)$.

The interaction of the CCSN shock with the upstream shear wave, characterized by the angle $\theta=\tan ^{-1}\left(k_{y} / k_{x}\right)$, is sketched in Fig. 4. As a result of the interaction, the shock ripples and the fluid downstream is correspondingly altered with acoustic and entropicvorticity waves, the former travelling at the speed of sound downstream $a_{2}$ and the latter moving with the fluid particles.

For the perturbed accretion shock to be seen as a discontinuity front, the characteristic perturbation wavelength $\lambda_{\mathrm{c}} \sim k_{y}^{-1}$ must be much larger than the accretion-shock thickness $l$, including the dissociation layer in it (see Fig. 1). Besides, to consider the baseflow variable as constant properties, the shock and the in-falling gas must be in the nearly steady regime, so that the variations of the base-flow properties within the characteristic wavelength can be neglected. These two conditions are simultaneously true for perturbation wavelengths much smaller than expanding shock evolution range and much higher than the shock thickness, both used to define the limits of validity of the model associated with spatial and temporal scales: $k_{y} R_{\text {shock }} \gg 1 \gg k_{y} l$ and $\dot{u}_{1} \ll k_{y} u_{1}^{2}$, respectively. 
On the other hand, the planar shock assumption, $k_{y} R_{\text {shock }} \gg 1$, is not suitable for perturbations characterized by low-mode numbers such as SASI (Blondin et al. 2003; Fernandez 2015). For such modes, spherical geometry is more suitable (Foglizzo 2009), which has been employed by Takahashi et al. (2016) to study the influence of pre-collapse perturbations on the hydrodynamic eigenmodes in the gain region.

For the analysis it is convenient to use a reference frame moving with the velocity of the post-shock flow. The solution is to be described in terms of the dimensionless coordinates $x=k_{y} x_{2}$ and $y=k_{y} y_{2}$ and the dimensionless time $\tau=a_{2} k_{y} t$.

The non-dimensional values for pressure, density, and velocity perturbations downstream, defined as

$\bar{p}=\frac{p-\left\langle p_{2}\right\rangle}{\gamma\left\langle p_{2}\right\rangle}, \quad \bar{\rho}=\frac{\rho-\left\langle\rho_{2}\right\rangle}{\left\langle\rho_{2}\right\rangle}$,

$\bar{u}=\frac{u-\left\langle u_{2}\right\rangle}{\left\langle a_{2}\right\rangle}, \quad \bar{v}=\frac{v-\left\langle v_{2}\right\rangle}{\left\langle a_{2}\right\rangle}$,

respectively, are used to write the adiabatic Euler equations governing the post-shock flow. Anticipating that $\bar{p}$ and $\bar{v}$ are always proportional to $\cos (y)$ and $\sin (y)$, respectively, the conservation equations for mass, $x$-momentum, $y$-momentum, and energy, namely

$$
\begin{gathered}
\frac{\partial \bar{\rho}}{\partial \tau}+\frac{\partial \bar{u}}{\partial x}+\bar{v}=0, \quad \frac{\partial \bar{u}}{\partial \tau}+\frac{\partial \bar{p}}{\partial x}=0, \\
\frac{\partial \bar{v}}{\partial \tau}-\bar{p}=0, \quad \frac{\partial \bar{p}}{\partial \tau}=\frac{\partial \bar{\rho}}{\partial \tau},
\end{gathered}
$$

respectively, are combined for $\bar{p}$ to yield

$$
\frac{\partial^{2} \bar{p}}{\partial \tau^{2}}=\frac{\partial^{2} \bar{p}}{\partial x^{2}}-\bar{p}
$$

as the two-dimensional periodically symmetric wave equation, which governs the perturbation field behind the shock.

The problem reduces to that of integrating the linearized Euler equations, or equivalently the wave equation (16), for $\tau \geq 0$ and within the domain delimited by the leading reflected sonic wave travelling backwards, $x=-\tau$ and the shock front moving upwards $x=M_{2} \tau$. One boundary condition is provided by the isolated shock assumption, which translates into not considering the effect of the acoustic waves reaching the shock front from behind, in consonance with the large radius limit, $R_{\text {shock }} k_{y} \gg 1$. On the other hand, the boundary condition at the CCSN shock is determined by the linearized Rankine-Hugoniot relationships,

$\left(C_{2}-1\right) \dot{\xi}_{\mathrm{s}}=C_{2} \bar{u}_{\mathrm{s}}-M_{2} C_{2} \bar{\rho}_{\mathrm{s}}-\bar{u}_{1}$

$\bar{p}_{\mathrm{s}}=2 M_{2}\left(\bar{u}_{\mathrm{s}}-\bar{u}_{1}\right)-M_{2}^{2} \bar{\rho}_{\mathrm{s}}$,

$M_{1}^{2} M_{2}^{2} \bar{\rho}_{\mathrm{s}}=\Pi_{\mathrm{s}} \bar{p}_{\mathrm{s}}-\Delta_{\mathrm{s}}\left(\dot{\xi}_{\mathrm{s}}-\bar{u}_{1}\right)$,

$\bar{v}_{\mathrm{s}}=M_{2}\left(C_{2}-1\right) \xi_{\mathrm{s}}+\bar{v}_{1}$,

with $\dot{\xi}_{\mathrm{s}}$ denoting the temporal derivative of the dimensionless ripple shock position $\xi_{\mathrm{s}}=k_{y}\left(x_{1, \mathrm{~s}}-u_{1} t\right)$, as depicted in Fig. 4.

The energy equation $(17 \mathrm{c})$, which involves the functions

$\Pi_{\mathrm{s}}=\frac{M_{1}^{2}\left[1+M_{1}^{2}(1-\kappa)\right]^{2}}{\left(M_{1}^{2}+1\right)^{2}-M_{1}^{4} \kappa^{2}}$

and

$\Delta_{\mathrm{s}}=\varepsilon \frac{2 M_{2}^{3} M_{1}^{6}(\gamma-1)\left[1+M_{1}^{2}(1-\kappa)\right]}{\left(M_{1}^{2}+1\right)^{2}-M_{1}^{4} \kappa^{2}}$, distinguishes regular adiabatic shocks from reacting shocks like detonations or nuclear-dissociating shocks.

In the previous work (Abdikamalov et al. 2016), the coefficients accompanying the linear perturbations in the linear energy equation $(17 \mathrm{c})$ were the same as those found in perturbed adiabatic shock $\left(\Pi_{\mathrm{s}}=1\right.$ and $\left.\Delta_{\mathrm{s}}=0\right)$, although the values of the baseflow properties, namely $M_{2}$ and $C_{2}$, were accordingly modified by nuclear dissociation effects. How nuclear dissociation degree is affected by the perturbations, and how that modification ultimately acts upon the downstream flow variables, is incorporated in this model through coefficients $\Pi_{\mathrm{s}}$ and $\Delta_{\mathrm{s}}$. In this sense, the present analysis consistently accounts for the effect of $\varepsilon$ in both zero-order and first-order flow variables.

The value of $\Pi_{\mathrm{s}}$ is positive when the dissociation energy is sufficiently low, that is $\kappa<1+M_{1}^{-2}$. On the other hand, when the dissociation energy is sufficiently high, the value of $\Pi_{\mathrm{s}}$ becomes negative, then reverting the relationship between density and pressure perturbations in equation (17c). Since the degree of dissociation depends on the shock strength, the term involving the function $\Delta_{\mathrm{s}}$ in equation $(17 \mathrm{c})$ is proportional to the incident Mach number perturbation $\delta M_{1}=\left(\dot{\xi}_{\mathrm{s}}-\bar{u}_{1}\right) M_{1} /\left(M_{2} C_{2}\right)$. The value of $\Delta_{\mathrm{s}}$ is found to be negative for $\varepsilon>0$. It is worth commenting that the case of exothermic detonations is significantly different since the second term in the right-hand side of equation (17c) will vanish (Huete, Sánchez \& Williams 2013; Huete et al. 2017). This is so because the total heat release, generated by the combustion process behind the shock, does not depend on the shock intensity perturbation, as it is provided by self-sustained reactions. Once the reaction is triggered it will release all the thermonuclear (or chemical) energy.

Algebraical manipulation of equations (17a)-(17d) is carried out to write one of the two equations for the shock boundary condition involving $\bar{\xi}_{\mathrm{s}}$ and $\bar{p}_{\mathrm{s}}$, that is

$$
\frac{\mathrm{d} \xi_{\mathrm{s}}}{\mathrm{d} \tau}=\sigma_{\mathrm{a}} \bar{p}_{\mathrm{s}}+\hat{u}_{1} \cos \left(\frac{k_{x}}{k_{y}} C_{2} M_{2} \tau\right),
$$

with the factor accompanying the pressure perturbation being

$\sigma_{\mathrm{a}}=\frac{C_{2}\left(M_{1}^{2}-\Pi_{\mathrm{s}}\right)}{2 M_{2} M_{1}^{2}\left(C_{2}-1\right)+C_{2} \Delta_{\mathrm{s}}}$.

Similarly, the material derivative behind the shock, $\partial /(\partial \tau)+$ $M_{2} \partial /(\partial x)$, of the streamwise velocity perturbation $\bar{u}_{\mathrm{s}}=\sigma_{\mathrm{b}} \bar{p}_{\mathrm{s}}+\bar{u}_{1}$, with

$\sigma_{\mathrm{b}}=\frac{M_{1}^{2}+\Pi_{\mathrm{s}}+\Delta_{\mathrm{s}} \sigma_{\mathrm{a}}}{2 M_{2} M_{1}^{2}}$,

is used to provide

$$
\begin{aligned}
\left(\sigma_{\mathrm{b}}+M_{2}\right) \frac{\partial \bar{p}_{\mathrm{s}}}{\partial \tau}+ & \left.\left(\sigma_{\mathrm{b}} M_{2}+1\right) \frac{\partial \bar{p}}{\partial x}\right|_{\mathrm{s}}=-M_{2}^{2}\left(C_{2}-1\right) \xi_{\mathrm{s}} \\
& +\frac{k_{x}}{k_{y}} M_{2}\left(C_{2}-1\right) \hat{u}_{1} \sin \left(\frac{k_{x}}{k_{y}} C_{2} M_{2} \tau\right)
\end{aligned}
$$

as the second equation that conforms, along with equation (20), the shock boundary condition for the functions $\bar{\xi}_{\mathrm{s}}$ and $\bar{p}_{\mathrm{s}}$.

The coefficients $\sigma_{\mathrm{a}}$ and $\sigma_{\mathrm{b}}$ are positive for any combination of parameters $M_{1}$ and $\varepsilon$. In the strong shock limit for $\varepsilon>0$, the value of $\sigma_{\mathrm{a}}$ approaches to zero with $\left.\sigma_{\mathrm{a}}\right|_{M_{1} \gg 1} \sim M_{1}^{-2}$, while $\sigma_{\mathrm{b}}$ reaches a constant value determined by the inverse of the post-shock Mach number, namely $\left.\sigma_{\mathrm{b}}\right|_{M_{1} \gg 1}=\left.M_{2}^{-1}\right|_{M_{1} \gg 1}$.

The initial condition of the shock perturbations is determined by knowing that the shock is initially planar, so that $\bar{\xi}_{\mathrm{s}}=\bar{v}_{\mathrm{s}}=0$. Correspondingly, the initial perturbations of pressure and streamwise 
velocity must satisfy $\bar{u}_{\mathrm{s}}+\bar{p}_{\mathrm{s}}=0$, as dictated by the first acoustic wave emitted backwards, thereby giving

$\bar{p}_{\mathrm{s} 0}=-\frac{1}{\sigma_{\mathrm{b}}+1} \hat{u}_{1}$

for the initial shock pressure perturbation.

\section{LINEAR INTERACTION ANALYSIS WITH MONOCHROMATIC VORTICITY PERTURBATIONS}

The description of the shock-turbulence encounter is simplified when the interaction with a single vorticity wave is addressed first. Both long-time and transient functions of the variables of interest will be described in this section, with the former being later employed in Section 4 to compute the shock effect on isotropic vorticity spectra. That is, as the transient time is found to be effectively short, the net effect of the shock on the turbulent field is then obtained by the linear superposition of the asymptotic long-time effect that the shock causes on every independent mode comprising the turbulence spectrum.

\subsection{Shock pressure and corrugation temporal evolution}

The asymptotic behaviour of the corrugated shock can be inferred from the Laplace transform expression provided in equation (A6), with the imaginary poles in the dispersion relationship

$$
\left(s \sqrt{s^{2}+1}+\sigma_{\mathrm{b}} s^{2}+\sigma_{\mathrm{c}}\right)\left(s^{2}+\zeta^{2}\right)=0,
$$

indicating the possibility of asymptotic harmonic oscillations. The left-hand product in equation (25) accounts for the shock response in the absence of continuous perturbations, whereas the right-hand product refers to the induced oscillations from the nonhomogeneous upstream flow. The characteristic dimensionless frequency $\zeta$ is provided in equation (27). Notice that the term $\sqrt{s^{2}+1}$ may change the sign if the pole lies on the bottom half-space of the imaginary plane.

It has been found that equation $s \sqrt{s^{2}+1}+\sigma_{\mathrm{b}} s^{2}+\sigma_{\mathrm{c}}=0$ has no poles, indicating that shock pressure perturbations decay with time in the absence of continuous excitement. Generally, the perturbations decay in time like $\tau^{-3 / 2}$, but this decay rate changes, however, for infinitely strong shocks with $\varepsilon=0$, since $\sigma_{\mathrm{b}}=\sigma_{\mathrm{c}}$, yielding $\tau^{-1 / 2}$ as the law describing the approach to the permanent solution (Fraley 1986).

It is convenient to study first the long-time response of the accretion shock to monofrequency perturbations. As $\sigma_{\mathrm{c}}<\sigma_{\mathrm{b}}$ the shock will oscillate only with the excitement frequency coming from upstream perturbations, $\omega_{\mathrm{s}}=R M_{2} k_{x} / k_{y}$, thereby yielding an asymptotic response qualitatively similar to the one found for adiabatic shock waves (Wouchuk et al. 2009)

$\bar{p}_{\mathrm{s}}(\tau \gg 1)= \begin{cases}\mathcal{P}_{\mathrm{lr}} \cos \left(\omega_{\mathrm{s}} \tau\right)+\mathcal{P}_{\mathrm{li}} \sin \left(\omega_{\mathrm{s}} \tau\right), & \zeta \leq 1, \\ \mathcal{P}_{\mathrm{s}} \cos \left(\omega_{\mathrm{s}} \tau\right), & \zeta \geq 1\end{cases}$

except for the coefficients defining the amplitudes, which are provided by equations (A15)-(A17) in Appendix A. As the planar infinitely thin assumption does not provide any length scale, the shock oscillation period will be proportional to the upstream characteristic length. In dimensional variables, the time between pressure peaks is given by $t_{\text {per }}=\lambda_{x} /\left(2 \pi a_{1} M_{1}\right)$.

As in previous LIA works (Wouchuk et al. 2009; Huete et al. 2013, 2017), the pressure perturbation field splits into two distinguished
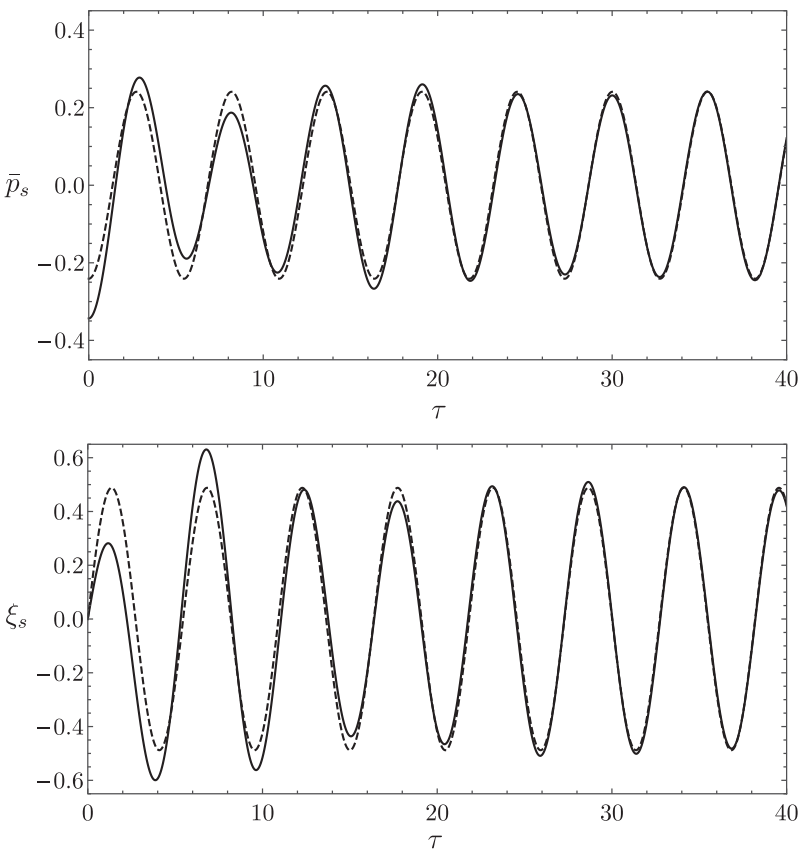

Figure 5. Shock pressure perturbation (top) and shock ripple amplitude (bottom) as a function of time $\tau$ for shock strength $M_{1}=5$, characteristic frequency $\zeta=1.2$, and for a dissociation energy parameter $\varepsilon=0.4$. Solid lines: transient evolutions (28) and (A14). Dashed lines: asymptotic longtime equations (26) and (A18).

regimes depending on the dimensionless frequency

$\zeta=\frac{k_{x}}{k_{y}} \frac{M_{2} C_{2}}{\sqrt{1-M_{2}^{2}}}=\frac{\omega_{\mathrm{s}}}{\sqrt{1-M_{2}^{2}}}$.

In the long-wavelength (low-frequency) regime, $\zeta<1$, the acoustic perturbation right behind the shock is composed by the amplitudes of two orthogonal contributions $\mathcal{P}_{\text {lr }}$, and $\mathcal{P}_{\text {li }}$, respectively. In this range, the amplitude of the pressure disturbances exponentially decays with the distance from the shock front. On the other hand, in the short-wavelength (high-frequency) regime $\zeta>1$, the acoustic radiation travels in the form of constant-amplitude waves. The critical value $\zeta=1$ then indicates the condition at which stable sonic perturbations downstream move parallel to the shock front in the shock reference frame.

As shown in equation (A9) of Appendix A, the post-shock pressure perturbation field can be computed as a linear combination of Bessel functions. In particular, right behind the shock, it is found that

$\bar{p}_{\mathrm{s}}(\tau)=\sum_{\nu=0}^{\infty} N_{\nu} J_{v}\left(r=\tau \sqrt{1-M_{2}^{2}}\right)$,

with the corresponding coefficients for $N_{v}$, provided in equation (A11), being obtained through the Laplace transform (A5) and the isolated shock boundary condition. The temporal evolution of the shock ripple $\xi_{\mathrm{s}}(\tau)$ is readily obtained through the integration of equation (20), whose solution can be expressed in terms of hypergeometrical functions, as shown in equation (A14). Akin to the shock pressure, the asymptotic long-time response is written in terms of harmonic functions, as provided in equation (A18).

The functions $\bar{p}_{\mathrm{s}}(\tau)$ and $\xi_{\mathrm{s}}(\tau)$ are computed in Fig. 5 as a function of $\tau$, for $M_{1}=5, \zeta=1.2$, and for $\varepsilon=0.4$. Both transient (solid line) and long-time response (dashed line) are shown. The shock transient evolution is found to agree fairly well with the asymptotic 
expressions provided in equations (26) and (A18), then confirming that asymptotic functions can be used to compute the interaction with an isotropic spectrum without significant loss of accuracy.

\subsection{Downstream flow variables}

The spatial distribution of the flow variables, namely, pressure, density, and velocity, is derived from the shock pressure evolution computed previously. For example, pressure perturbations downstream can be written in terms of Bessel functions as

$\bar{p}(x, \tau)=\sum_{\nu=0}^{\infty} N_{\nu} J_{\nu}\left(\sqrt{\tau^{2}-x^{2}}\right) \mathrm{e}^{-v\left[\tanh ^{-1}\left(M_{2}\right)-\tanh ^{-1}\left(\frac{x}{\tau}\right)\right]}$.

As the asymptotic expression (26) is found to reproduce accurately the shock pressure evolution, the asymptotic long-time response of the shock is employed to compute the post-shock disturbances.

Downstream linear perturbations are conveniently split into entropic-vortical, conveyed by the fluid particles, and travelling acoustic modes (Kovasznay 1953; Chu \& Kovasznay 1957), namely

$\bar{p}(x, \tau)=\bar{p}_{\mathrm{a}}(x, \tau), \quad \bar{\rho}(x, \tau)=\bar{\rho}_{\mathrm{a}}(x, \tau)+\bar{\rho}_{\mathrm{e}}(x)$,

$\bar{u}(x, \tau)=\bar{u}_{\mathrm{a}}(x, \tau)+\bar{u}_{\mathrm{r}}(x), \quad \bar{v}(x, \tau)=\bar{v}_{\mathrm{a}}(x, \tau)+\bar{v}_{\mathrm{r}}(x)$.

In the absence of diffusive effects, the amplitudes of the entropic solenoidal perturbations are given by their corresponding values generated right behind the shock, and they are steady in a reference frame comoving with the fluid particles. Acoustic disturbances, on the other hand, refer to travelling sonic waves that escape from the shock when $\zeta>1$.

The acoustic radiation condition is then determined by $\omega_{\mathrm{s}}>$ $\left(1-M_{2}^{2}\right)^{1 / 2}$, a condition that depends on the upstream shear wave, since $\zeta=[0, \infty)$ depends on the relative properties of the perturbation field ahead of the shock. Small values of $\zeta$ represent the interaction with upstream vortices highly stretched in the streamwise direction $\lambda_{x} \gg \lambda_{y}$, while the opposite is true for $\zeta \gg 1$. In the latter low mode-number scenario $\left(\lambda_{x} \ll \lambda_{y}\right)$, the problem reduces to the one-dimensional interaction of the shock with radial perturbation waves. Such stability analysis has been developed by Velikovich et al. (2016) for the classical Noh's configuration in adiabatic conditions. The asymptotic far-field solution for the acoustic disturbances is also written in terms of harmonic functions, representing stable travelling fronts that occur only when the shock oscillation frequency is sufficiently high, $\zeta>1$.

Travelling sonic perturbations are functions of $\left(\omega_{\mathrm{a}} \tau-k_{\mathrm{a}} x\right)$, with the frequency $\omega_{\mathrm{a}}$ and the wavenumber $k_{\mathrm{a}}$ being determined by the post-shock adiabatic dispersion relationship $\omega_{\mathrm{a}}^{2}=k_{\mathrm{a}}^{2}+1$, and the shock oscillation frequency $\omega_{\mathrm{s}}=\omega_{\mathrm{a}}-M_{2} k_{\mathrm{a}}$, yielding

$\omega_{\mathrm{a}}=\frac{\omega_{\mathrm{s}}-M_{2} \sqrt{\omega_{\mathrm{s}}^{2}-1+M_{2}^{2}}}{1-M_{2}^{2}}$

and

$k_{\mathrm{a}}=\frac{\omega_{\mathrm{s}} M_{2}-\sqrt{\omega_{\mathrm{s}}^{2}-1+M_{2}^{2}}}{1-M_{2}^{2}}$,

respectively, which depend upon the shock frequency $\omega_{\mathrm{s}}$. It is straightforward to see that $k_{\mathrm{a}}$ can be either negative or positive, the former representing the sonic waves propagating downwards in the compressed gas reference frame, and the latter denoting the waves moving upwards, although never catching up the shock wave as dictated by the isolated front boundary condition. The shock oscillation frequency, $\omega_{\mathrm{s}}=1$, marks the standing acoustic wave regime, therefore separating the left travelling solution $\omega_{\mathrm{s}}>1$ from the right travelling regime $\left(1-M_{2}^{2}\right)^{1 / 2}<\omega_{\mathrm{s}}<1$ in the compressed gas reference frame. When the shock oscillates with two frequencies, the possibility of having sonic fronts running upstream and downstream is possible.

The asymptotic pressure and isentropic density perturbations, far behind the shock, equal

$\bar{p}(x, \tau)=\bar{\rho}_{\mathrm{a}}(x, \tau)=\mathcal{P}_{\mathrm{s}} \cos \left(\omega_{\mathrm{a}} \tau-k_{\mathrm{a}} x\right)$,

with $\mathcal{P}_{\mathrm{s}}$ standing for the amplitude of the shock pressure disturbances in the long-wavelength regime. The amplitude of the associated acoustic velocity perturbations is proportional to the pressure changes through the functions (A23), provided in Appendix A. The corresponding isentropic temperature variations induced by the acoustic shock radiation are simply $\bar{T}_{\mathrm{a}}(x, \tau)=(\gamma-1) \bar{p}(x, \tau)$.

The entropic contribution to the density perturbations $\bar{\rho}_{\mathrm{e}}$ is computed from Rankine-Hugoniot relations (17a)-(17c), after subtracting the acoustic part. It is readily seen that

$\bar{\rho}_{\mathrm{e}}(x)=(\mathcal{D}-1) \bar{p}_{\mathrm{s}}\left(\tau=\frac{x}{M_{2}}\right)$,

with $\mathcal{D}=\left(2 M_{2} \sigma_{\mathrm{b}}-1\right) / M_{2}^{2}$ being the amplitude of the density perturbations behind the shock. As easily inferred from Fig. 2, the value of $\mathcal{D}$ is found to be positive and reaches a constant value in the strong-shock limit given by $\left.\mathcal{D}\right|_{M_{1} \gg 1}=2 M_{2}^{-2}$. The corresponding isobaric temperature perturbation, scaled with base-flow temperature, is the function $\bar{T}_{\mathrm{e}}(x)=-\bar{\rho}_{\mathrm{e}}(x)=-(\mathcal{D}-1) \bar{p}_{\mathrm{s}}\left(\tau=x / M_{2}\right)$.

Analogously, dimensionless vorticity disturbances are determined by

$\bar{\omega}(x)=\frac{\partial \bar{v}}{\partial x}-\frac{\partial \bar{u}}{\partial y}=\Omega_{2} \bar{p}_{\mathrm{s}}\left(\tau=\frac{x}{M_{2}}\right)+\Omega_{1} \cos \left(\frac{\omega_{\mathrm{s}}}{M_{2}} x\right)$,

with

$\Omega_{1}=C_{2}\left[1+\left(\frac{k_{x}}{k_{y}}\right)^{2}\right]=C_{2}\left(1+\frac{1-M_{2}^{2}}{C_{2}^{2} M_{2}^{2}} \zeta^{2}\right)$

indicating the contribution result of the one-dimensional compression effect, the shrinking of the vortices by the overall mass compression ratio, and

$\Omega_{2}=\frac{M_{2}\left(C_{2}-1\right) \sigma_{\mathrm{a}}+\sigma_{\mathrm{b}} M_{2}-1}{M_{2}}$

referring to contribution induced by shock rippling proportional to pressure, a two-dimensional effect.

The rotational contribution for the velocity disturbances is readily computed through the vorticity field, by knowing that rotational perturbations are steady and isobaric in the linear inviscid approach. The relationships

$\bar{\omega}(x)=-\frac{\partial^{2} \bar{u}_{\mathrm{r}}}{\partial x^{2}}+\bar{u}_{\mathrm{r}} \quad$ and $\quad \bar{v}_{\mathrm{r}}(x)=-\frac{\partial \bar{u}_{\mathrm{r}}}{\partial x}$

are then employed to write the asymptotic longitudinal and transverse rotational velocity distributions, provided in equations (A24) and (A25).

The asymptotic expressions for the rotational velocity and entropic density perturbations are computed in Fig. 6, for the same conditions as in Fig. 5. Velocity perturbations are displayed in a two-dimensional vector field, with the length of the vectors being scaled within the maximum and minimum velocity amplitudes, 1.9 and 0.45 , respectively. Transverse component of the velocity perturbations is found to be much greater than longitudinal contribution. 


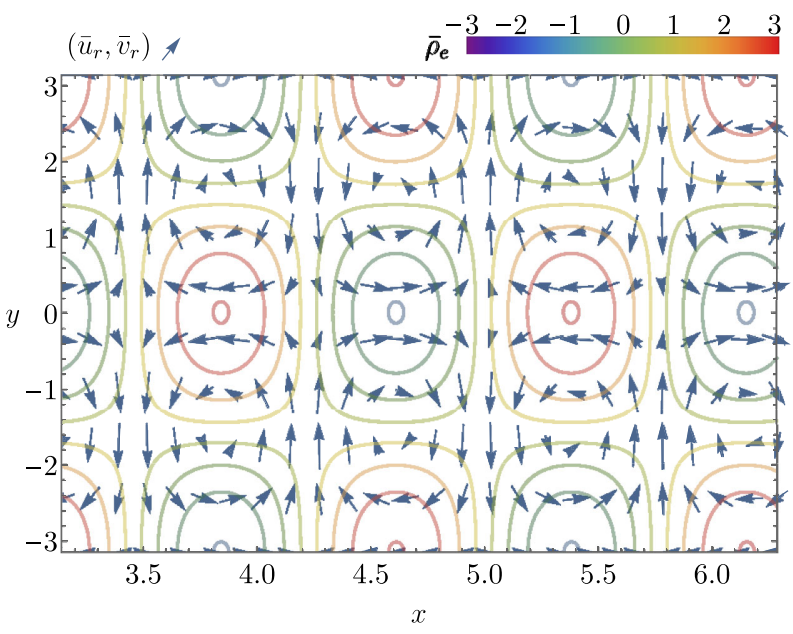

Figure 6. Two-dimensional vector field plot for rotational velocity perturbations superposed to isocontours of entropic density disturbances for a shock wave with shock intensity $M_{1}=5$, dissociation energy parameter $\varepsilon=0.4$, and characteristic frequency $\zeta=1.2$.

The spatial frequency modulation, given by $\omega_{\mathrm{s}} / M_{2}$ is clearly distinguished. The amplitude of the rotational perturbations depends on the incident angle $\theta$, as shown in Fig. A1 for $M_{1}=5$. This dependence is later used to account for the interaction with a whole spectrum of vorticity waves, with $\theta$ ranging from 0 to $\pi$, upon consideration of the isotropic probability distribution.

Superposed to the vector field, the entropic density disturbances are displayed in a contour plot in Fig. 6. The centre of the eddies and the peaks of the density field are shifted in $\pi / 2$ in the lateral coordinate, as the former are proportional to $\sin (y)$ and the second one to $\cos (y)$. The position of the peak values of density and rotational perturbations are in phase for $\zeta>1$ along the streamwise direction, as both periodic distributions are proportional to $\cos \left(\omega_{\mathrm{s}} / M_{2} x\right)$. There exists a spatial shift between the rotational and entropic mode, $\Delta \phi=\phi_{\mathrm{r}}-\phi_{\mathrm{e}}$, for $\zeta<1$, given by the contribution of the orthogonal components, $\tan \phi_{\mathrm{r}}=\Omega_{2} \mathcal{P}_{\mathrm{li}} /\left(\Omega_{2} \mathcal{P}_{\mathrm{lr}}+\Omega_{1}\right)$ and $\tan \phi_{\mathrm{e}}=\mathcal{P}_{\mathrm{li}} / \mathcal{P}_{\mathrm{lr}}$.

\section{LINEAR INTERACTION ANALYSIS WITH 3D ISOTROPIC VORTICITY PERTURBATIONS}

As critical conditions for CCSN explosion have been found to be sensitive to turbulent fluctuations, the amplification of average perturbations conveyed by the in-falling gas across the shock is addressed in this section. In particular, amplification ratios for turbulent kinetic energy, enstrophy, and turbulent Mach number are computed as a function of the governing 1D parameters: shock strength and the nuclear dissociation energy.

\subsection{Turbulent kinetic energy}

The 3D upstream flow is assumed to be homogeneous and isotropic. Therefore, the amplitude of the incident shear wave $\hat{u}_{1}$ depends exclusively on the wavenumber amplitude $|\boldsymbol{k}|=k$ as $\boldsymbol{k}$ is uniformly distributed over the unit sphere. The $3 \mathrm{D}$ problem is conveniently formulated in spherical polar coordinates, so the upstream velocity field $\left(\bar{u}_{1}, \bar{v}_{1}, \bar{w}_{1}\right)=\hat{u}_{1}(\sin \theta \sin \varphi, \cos \theta \sin \varphi, \cos \varphi)$ and the associated wavenumber vector is $k=k(\cos \theta,-\sin \theta, 0)$. The interaction with the whole spectrum of perturbations is carried out by direct superposition of linear perturbations (Batchelor 1953). The average upstream velocity perturbation is

$$
\begin{aligned}
& \left\langle\bar{u}_{1}^{2}\right\rangle=\int_{k^{3}}\left|\bar{u}_{1}\right|^{2} \mathrm{~d} k^{3}=\frac{8 \pi}{3} \int_{0}^{\infty} \hat{u}_{1}^{2}(k) k^{2} \mathrm{~d} k, \\
& \left\langle\bar{v}_{1}^{2}\right\rangle=\left\langle\bar{w}_{1}^{2}\right\rangle=\int_{k^{3}}\left|\bar{v}_{1}\right|^{2} \mathrm{~d} k^{3}=\frac{2 \pi}{3} \int_{0}^{\infty} \hat{u}_{1}^{2}(k) k^{2} \mathrm{~d} k,
\end{aligned}
$$

so the corresponding turbulent kinetic energy (TKE) computes as

$\mathrm{TKE}_{1}=\frac{1}{2}\left(\left\langle\bar{u}_{1}^{2}\right\rangle+\left\langle\bar{v}_{1}^{2}\right\rangle+\left\langle\bar{w}_{1}^{2}\right\rangle\right)=2 \pi \int_{0}^{\infty} \hat{u}_{1}^{2}(k) k^{2} \mathrm{~d} k$,

with $\hat{u}_{1}(k)=$ fun $(k)$ representing the isotropic energy spectrum.

The problem is further simplified by reducing the 3D geometry into an equivalent two-dimensional case that accounts for the effect of vorticity perturbations that are parallel or perpendicular to the shock propagation velocity. After some straightforward algebra, the amplification ratio across the shock wave is

$K=\frac{\mathrm{TKE}_{2}}{\mathrm{TKE}_{1}}=\frac{1}{2} \int_{0}^{\pi / 2}\left(\bar{u}^{2}+\bar{v}^{2}\right) \sin ^{3} \theta \mathrm{d} \theta+\frac{1}{2}$,

which is conveniently rewritten in terms of the integration variable $\zeta$ as

$K=\frac{1}{3} \int_{0}^{\infty}\left(\bar{u}^{2}+\bar{v}^{2}\right) P(\zeta) \mathrm{d} \zeta+\frac{1}{2}$,

with

$P(\zeta)=\frac{3}{2} \frac{M_{2}^{4} C_{2}^{4} \sqrt{1-M_{2}^{2}}}{\left[M_{2}^{2} C_{2}^{2}+\zeta^{2}\left(1-M_{2}^{2}\right)\right]^{5 / 2}}$

standing for the normalized probability-density distribution obeying $\int_{0}^{\infty} P(\zeta) \mathrm{d} \zeta=1$. It is readily seen that, although post-shock turbulence spectrum depends on upstream energy distribution $\int_{0}^{\infty} \hat{u}_{1}^{2}(k) k^{2} \mathrm{~d} k$, the kinetic energy amplification ratio does not as long as isotropic conditions are considered, namely $\hat{u}_{1}(k)=f u n(k)$.

The amplification ratios for the longitudinal and transverse kinetic energy contributions can be computed with the aid of the probability density distribution. They are conveniently split into rotational and acoustic contributions, yielding

$$
\begin{aligned}
L= & L_{\mathrm{r}}+L_{\mathrm{a}}=\int_{0}^{1}\left[\left(\mathcal{U}_{\mathrm{li}}^{\mathrm{r}}\right)^{2}+\left(\mathcal{U}_{\mathrm{li}}^{\mathrm{r}}\right)^{2}\right] P(\zeta) \mathrm{d} \zeta \\
& +\int_{1}^{\infty}\left(\mathcal{U}_{\mathrm{s}}^{\mathrm{r}}\right)^{2} P(\zeta) \mathrm{d} \zeta+\int_{1}^{\infty}\left(\mathcal{U}^{\mathrm{a}}\right)^{2} P(\zeta) \mathrm{d} \zeta
\end{aligned}
$$

for the longitudinal part. The variation of the velocity perturbation amplitudes with $\zeta$ is deduced from Fig. A1 (for $M_{1}=5$ ) knowing that $\zeta$ is inversely proportional to $\tan \theta$, as equation (27) reads.

Equivalently, the turbulent kinetic energy associated with the transverse contribution is

$$
\begin{aligned}
T= & T_{\mathrm{r}}+T_{\mathrm{a}}=\frac{1}{2} \int_{0}^{1}\left[\left(\mathcal{V}_{\mathrm{li}}^{\mathrm{r}}\right)^{2}+\left(\mathcal{V}_{\mathrm{li}}^{\mathrm{r}}\right)^{2}\right] P(\zeta) \mathrm{d} \zeta \\
& +\frac{1}{2} \int_{1}^{\infty}\left(\mathcal{V}_{\mathrm{s}}^{\mathrm{r}}\right)^{2} P(\zeta) \mathrm{d} \zeta+\frac{1}{2} \int_{1}^{\infty}\left(\mathcal{V}^{\mathrm{a}}\right)^{2} P(\zeta) \mathrm{d} \zeta+\frac{3}{4} .
\end{aligned}
$$

The total turbulent kinetic energy, also split into rotational and acoustic contributions through $K=K_{\mathrm{r}}+K_{\mathrm{a}}$, is computed with the aid of $K_{\mathrm{r}}=\left(L_{\mathrm{r}}+2 T_{\mathrm{r}}\right) / 3$ and $K_{\mathrm{a}}=\left(L_{\mathrm{a}}+2 T_{\mathrm{a}}\right) / 3$, or equivalently through $K=(L+2 T) / 3$.

The variation of the longitudinal, transverse, and total contributions for the turbulent kinetic energy is shown in Fig. 7 as a function of $M_{1}$, for $\varepsilon=0,0.2$, and 0.4 . The solid lines show the rotational contribution, and the dashed lines include the contribution of both 

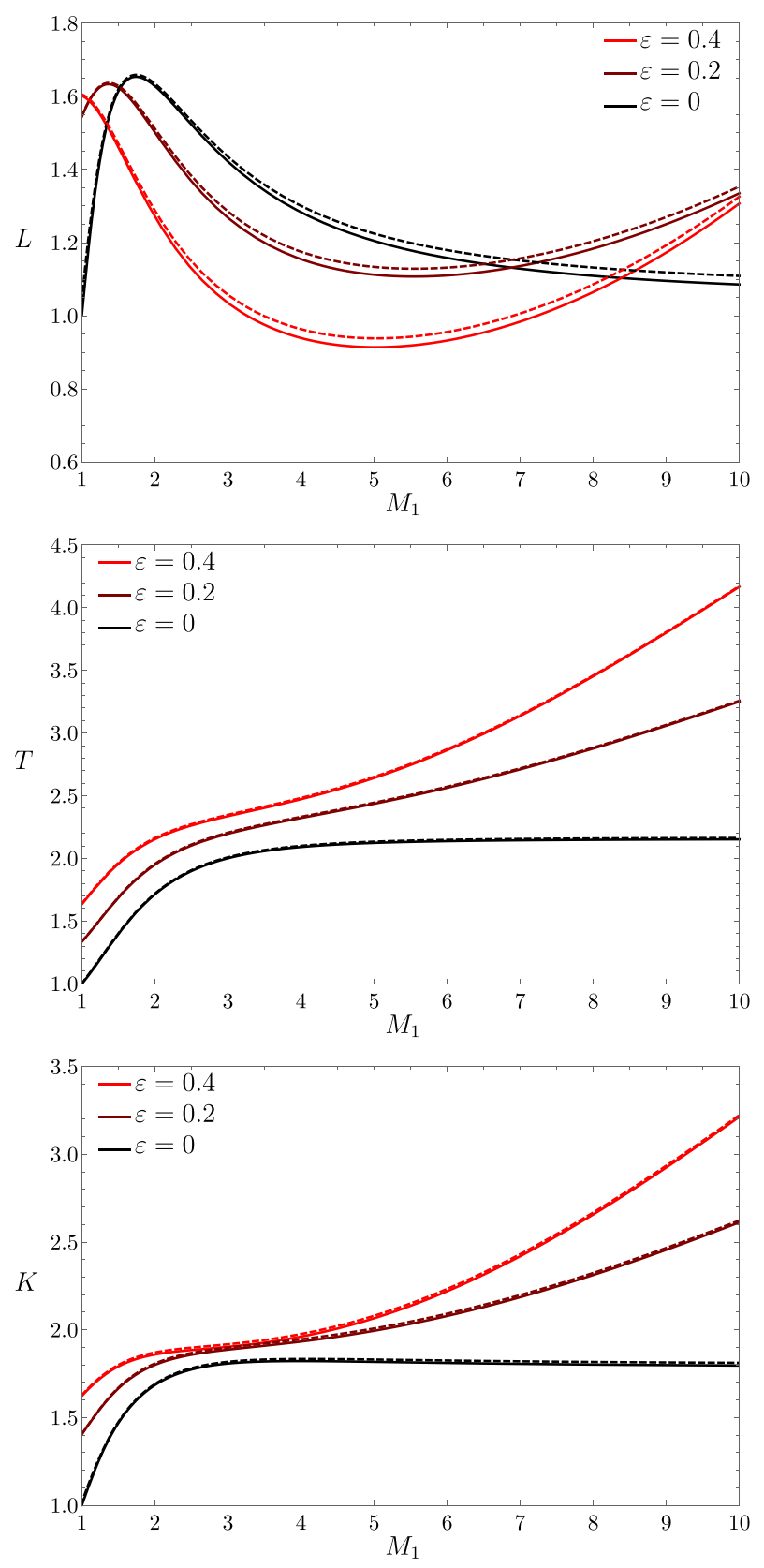

Figure 7. Longitudinal $L$, transverse $T$, and total $K$ kinetic energy amplification factors for dissociation energy parameters $\varepsilon=0,0.2$, and 0.4 . The solid lines show the rotational contribution, and the dashed lines account for both rotational and acoustic contributions.

rotational and acoustic kinetic energy. In agreement with Fig. A1, the acoustic contribution is found to be greater for the longitudinal part $L$, although sufficiently small to be neglected for any $M_{1}$ and $\varepsilon$ considered. Although not clearly seen in Fig. 7, the function $K$ approaches a constant value in the strong shock limit $M_{1} \gg 1$, they are $1.8,7.1$, and 9.8 for $\varepsilon=0,0.2$, and 0.4 , respectively. On the other hand, the weak shock limit $M_{1}-1 \ll 1$ provides $1,1.4$, and 1.6 for the same conditions. For a fixed value of the incident Mach number, the effect of nuclear dissociation is seen to increase the total kinetic energy. It is found that, for a Mach number close to 3, the total kinetic energy is less sensitive to dissociation energy, although longitudinal and transverse contributions are clearly counteraffected. It indicates that post-shock anisotropy is modified by $\varepsilon$. Longitudinal

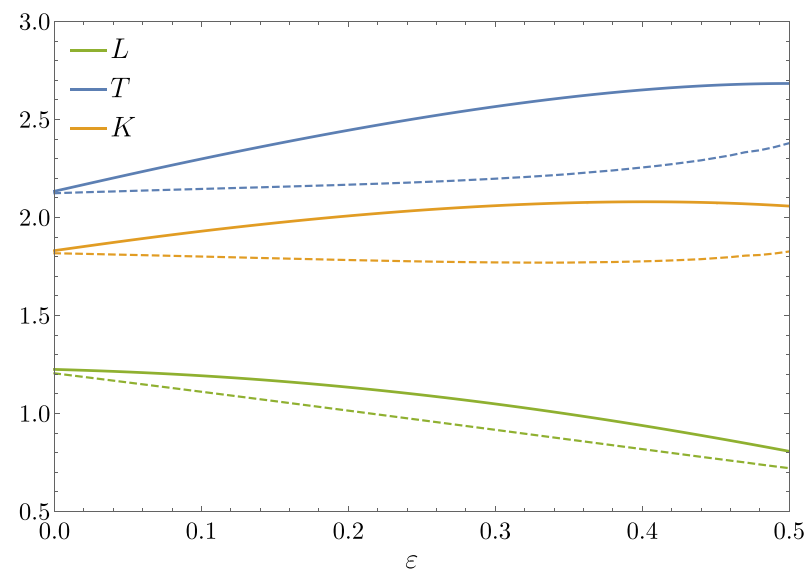

Figure 8. Longitudinal $L$, transverse $T$, and total $K$ kinetic energy amplification factors for a shock intensity $M_{1}=5$ as a function of the dissociation energy parameter $\varepsilon$. Solid lines represent computations of equations (44), (45) and (42) for $L, T$, and $K$, respectively, while dashed lines show the predictions in Abdikamalov et al. (2016).

contribution is generally diminished by nuclear dissociation if the Mach number is sufficiently high, a region that covers the scenarios of most interest. It is also found that transverse perturbations across the shock are more sensitive to the shock passage, then conforming a post-shock flow that differs from the ideal 1D configuration.

A direct comparison with the results obtained in Abdikamalov et al. (2016) reveals that the dependence of the turbulent kinetic energy with $M_{1}$ and $\varepsilon$ is affected when endothermic effects are included in the linear perturbation analysis. Although similar trends, when increasing $\varepsilon$, is found in both works, the values may differ substantially when the energy employed in dissociating the gas is sufficiently high. For the sake of exemplification, predictions for $L, T$, and $K$ are computed in Fig. 8 by using equations (44), (45), and (42) (solid) and recasting the data in Abdikamalov et al. (2016) (dashed). The differences become more pronounced with increasing shock strength, reaching $\sim 30$ per cent in $K$ for $M_{1}=10$ and $\varepsilon=0.4$.

\subsection{Turbulent Mach number}

It is instructive to relate the pre-shock and post-shock turbulent Mach numbers. It is immediate to see that

$\left\langle\delta M_{2}^{2}\right\rangle=-4 M_{2}\langle\bar{u} \bar{a}\rangle+\left\langle\bar{u}^{2}\right\rangle+\left\langle\bar{v}^{2}\right\rangle+\left\langle\bar{w}^{2}\right\rangle+3 M_{2}^{2}\left\langle\bar{a}^{2}\right\rangle$,

which can be split into entropic-rotational and acoustic contributions as $\left\langle\delta M_{2}^{2}\right\rangle=\left\langle\delta M_{1}^{2}\right\rangle\left(\Phi_{\mathrm{er}}+\Phi_{\mathrm{ac}}\right)$ in terms of the turbulent Mach number transference functions, $\Phi_{\mathrm{er}}$ and $\Phi_{\mathrm{ac}}$, for the entropic and acoustic parts, respectively. For isotropic turbulence in the upstream flow, the entropic-rotational part reads

$$
\begin{aligned}
\Phi_{\mathrm{er}} & =\frac{M_{2}^{2} C_{2}^{2}}{M_{1}^{2}}\left[\frac{\left\langle\bar{u}_{\mathrm{r}}^{2}\right\rangle+\left\langle\bar{v}_{\mathrm{r}}^{2}\right\rangle+\left\langle\bar{w}_{\mathrm{r}}^{2}\right\rangle}{3\left\langle\bar{u}_{1}^{2}\right\rangle}+\frac{M_{2}^{2}}{4} \frac{\left\langle\bar{\rho}_{\mathrm{e}}^{2}\right\rangle}{\left\langle\bar{u}_{1}^{2}\right\rangle}+\frac{2 M_{2}}{3} \frac{\left\langle\bar{u}_{\mathrm{r}} \bar{\rho}_{\mathrm{e}}\right\rangle}{\left\langle\bar{u}_{1}^{2}\right\rangle}\right] \\
& =\frac{M_{2}^{2} C_{2}^{2}}{M_{1}^{2}}\left[K_{\mathrm{r}}+\frac{M_{2}^{2}}{4} D_{\mathrm{e}}+\frac{2 M_{2}}{3} B_{\mathrm{er}}\right],
\end{aligned}
$$

while the acoustic contribution can be expressed as

$$
\begin{aligned}
\Phi_{\mathrm{ac}} & =\frac{M_{2}^{2} C_{2}^{2}}{M_{1}^{2}}\left[\frac{\left\langle\bar{u}_{\mathrm{a}}^{2}\right\rangle+\left\langle\bar{v}_{\mathrm{a}}^{2}\right\rangle}{3\left\langle\bar{u}_{1}^{2}\right\rangle}+\frac{M_{2}^{2}}{4} \frac{\left\langle\bar{\rho}_{\mathrm{a}}^{2}\right\rangle}{\left\langle\bar{u}_{1}^{2}\right\rangle}-\frac{2 M_{2}}{3} \frac{\left\langle\bar{u}_{\mathrm{a}} \bar{\rho}_{\mathrm{a}}\right\rangle}{\left\langle\bar{u}_{1}^{2}\right\rangle}\right] \\
& =\frac{M_{2}^{2} C_{2}^{2}}{M_{1}^{2}}\left[K_{\mathrm{a}}+\frac{M_{2}^{2}(\gamma-1)^{2}}{4} D_{\mathrm{a}}-\frac{2 M_{2}(\gamma-1)}{3} B_{\mathrm{a}}\right] .
\end{aligned}
$$




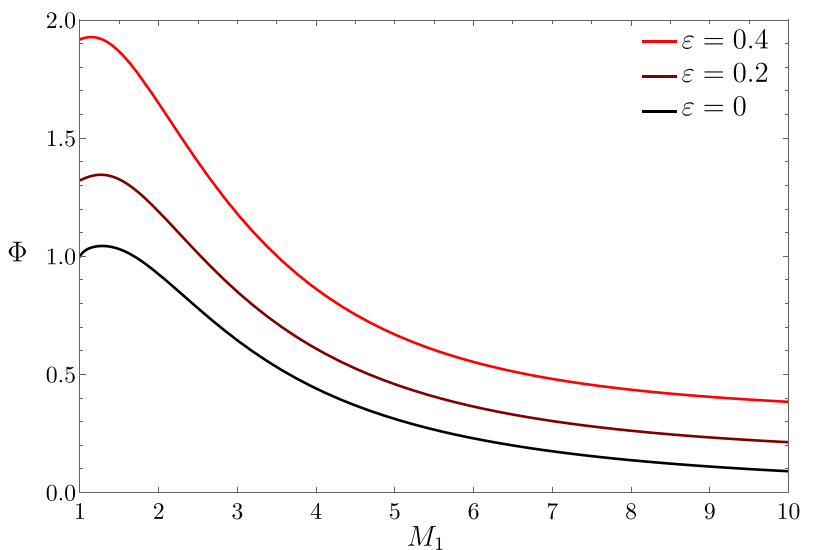

Figure 9. Turbulent Mach number transference function $\Phi$ as a function of the shock strength $M_{1}$ and for dissociation energy parameters $\varepsilon=0,0.2$, and 0.4 .

The values of $K_{\mathrm{r}}, K_{\mathrm{a}}, D_{\mathrm{e}}, D_{\mathrm{a}}, B_{\mathrm{er}}$, and $B_{\mathrm{a}}$ are provided in equation (42) for the kinetic energy, in equation (58) for the average density perturbations, and in equation (64) for the buoyancy correlation. The mean value of the post-shock Mach number includes changes in the velocity field, density, and the cross-product contribution. As $\bar{v}$ and $\bar{\rho}$ are orthogonal functions, only the longitudinal contribution correlates with density perturbations. The latter are being expressed as a function of shock pressure through $\bar{\rho}_{\mathrm{e}}(x)=(\mathcal{D}-1) \bar{p}_{\mathrm{s}}\left(\tau=x / M_{2}\right)$ for then entropic perturbations, and through $\bar{\rho}_{\mathrm{a}}=\bar{p}_{\mathrm{s}}\left(\tau=x / M_{2}\right)$ for the acoustic part.

The value of turbulent Mach number transference function $\Phi=\Phi_{\mathrm{er}}+\Phi_{\mathrm{ac}}$ is computed in Fig. 9 as a function of the shock strength $M_{1}$ for $\varepsilon=0,0.2$, and 0.4 . For typical values these parameters $\left(0.2 \lesssim \varepsilon \lesssim 0.4\right.$ and $\left.M_{1} \gtrsim 5\right), \Phi$ ranges from $\sim 0.3$ to $\sim 0.6$. Similarly to the turbulent kinetic energy in the post-shock region, most of the contribution to $\Phi$ comes from the entropic-rotational part, while the acoustic contribution $\Phi_{\mathrm{ac}}$ is found to be negligibly small.

\subsection{Enstrophy}

The effect of the shock passage on the upstream isotropic vorticity field can be computed with the aid of equations (34) and (43). The amplification of the average squared vorticity perturbations, non-dimensionalized with $\left(k a_{2}\right)^{2}$, is written as

$W=\frac{\left\langle\bar{\omega}_{x}^{2}+\bar{\omega}_{y}^{2}+\bar{\omega}_{z}^{2}\right\rangle}{\left\langle\bar{\omega}_{1, x}^{2}+\bar{\omega}_{1, y}^{2}+\bar{\omega}_{1, z}^{2}\right\rangle}=\frac{1}{3}+\frac{2}{3} \frac{\left\langle\bar{\omega}_{y}^{2}+\bar{\omega}_{z}^{2}\right\rangle}{\left\langle\bar{\omega}_{1, y}^{2}+\bar{\omega}_{1, z}^{2}\right\rangle}=\frac{1}{3}+\frac{2}{3} W_{\perp}$,

with the factor $1 / 3$ referring to the invariable component of the vorticity pointing in the streamwise direction, and $W_{\perp}$ being the amplification factor of the averaged squared vorticity perpendicular to the shock propagation velocity. The two-dimensional equivalent factor

$$
\begin{aligned}
W_{z}= & \frac{\left\langle\bar{\omega}_{z}^{2}\right\rangle}{\left\langle\bar{\omega}_{1, z}^{2}\right\rangle}=\int_{1}^{\infty}\left(\Omega_{1}+\Omega_{2} \mathcal{P}_{\mathrm{s}}\right)^{2} \frac{C_{2}^{2} M_{2}^{2}}{C_{2}^{2} M_{2}^{2}+\left(1-M_{2}^{2}\right) \zeta^{2}} P(\zeta) \mathrm{d} \zeta \\
& +\int_{0}^{1}\left[\left(\Omega_{1}+\Omega_{2} \mathcal{P}_{\mathrm{lr}}\right)^{2}+\Omega_{2}^{2} \mathcal{P}_{\mathrm{li}}^{2}\right] \frac{C_{2}^{2} M_{2}^{2}}{C_{2}^{2} M_{2}^{2}+\left(1-M_{2}^{2}\right) \zeta^{2}} P(\zeta) \mathrm{d} \zeta
\end{aligned}
$$

is conveniently employed in computing the perpendicular contribution as $W_{\perp}=\left(C_{2}+3 W_{z}\right) / 4$.

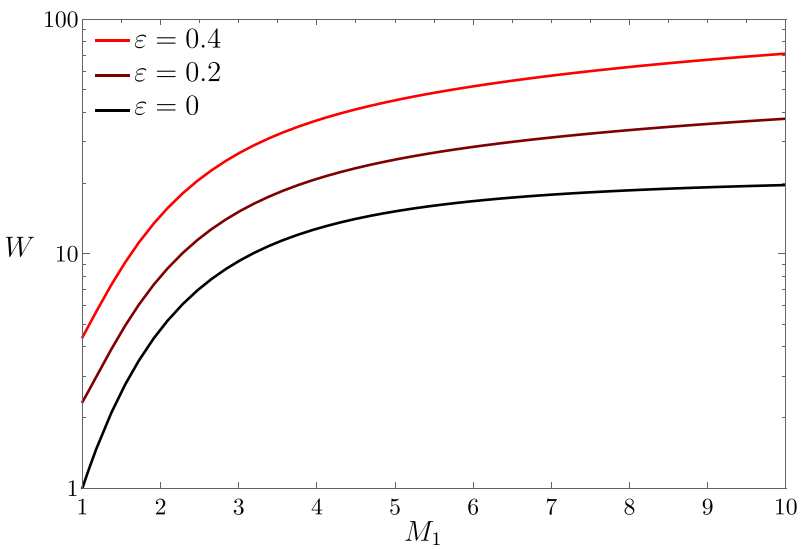

Figure 10. Amplification factor of the mean squared vorticity perturbations, $W$, as a function of the shock strength $M_{1}$ and for dissociation energy parameters $\varepsilon=0,0.2$, and 0.4 .

The so-called enstrophy, $W$, is computed in Fig. 10 for the same conditions as in Fig. 7. In consonance to the turbulent kinetic energy, the effect of nuclear dissociation across the shock is found to increase the average vorticity intensity, for a fixed value of $M_{1}$.

When the shock is expanding at variable Mach number, the theory still holds if base-flow changes are negligible within the perturbation wavelength distance. Upstream turbulent flows characterized by short wavelengths will meet this constriction. On the other side, perturbations must be sufficiently large for the shock to be seen as a pure discontinuity. In such case, the post-shock kinetic energy at any radial locus can be approximated by the one left by the expanding shock, whose instantaneous properties $M_{1}$ and $\varepsilon$ can be computed following the analysis presented in next section. The values obtained for the downstream kinetic energy and enstrophy can be then used to compute the evolution of the turbulent flow by viscous-dissipative and buoyant effects. Then, Figs 7 and 10 serve as the onset for such post-shock stage, with the subsequent thermalization of the kinetic energy being inferred by the dissipation energy cascade associated with the dominant scales (Mabanta \& Murphy 2017).

\section{NUCLEAR DISSOCIATION ENERGY AND THE PRE-SHOCK MACH NUMBER IN CCSN MODELS}

This section presents the estimates of the nuclear dissociation energy and the pre-shock Mach number from a series of spherically symmetric CCSN simulations using the GR1D code with the leakage/heating scheme (O'Connor \& Ott 2010). Eight Woosley \& Heger (2007) progenitor star models with ZAMS masses of 12 , $15,18,20,25,30,40$, and $70 \mathrm{M}_{\odot}$ were considered. Each progenitor model is evolved using several values of the heating parameter. This yields a variety of qualitatively different evolutionary paths for each stellar model, ranging from non-exploding models to rapidly exploding models. Each simulation is named using the following convention: for example, the simulation $s 15 h 1.23$ uses a progenitor model with a ZAMS mass of $15 \mathrm{M}_{\odot}$ evolved with heating factor of 1.23 (for the definition of the heating factor, see e.g. O'Connor \& Ott 2010; Ott et al. 2013).

The simulations use the SFHo finite-temperature nuclear equation of state (EOS) of Steiner, Hempel \& Fischer $(2013)^{1}$ as this

\footnotetext{
${ }^{1}$ Available at www.stellarcollapse.org (O’Connor \& Ott 2010).
} 
EOS employs an accurate treatment of light nuclei. Calculations with the Lattimer \& Douglas Swesty (1991) EOS with nuclear incompressibility of $K=220 \mathrm{MeV}$ revealed similar results. Across the computational domain it is used 1000 logarithmic radial grids with the central resolution of $0.1 \mathrm{~km}$. The outer boundary is fixed at the radius where the initial density is $2 \times 10^{3} \mathrm{~g} \mathrm{~cm}^{-3}$.

The shock wave dissociates heavy nuclei into light nuclei such as $\alpha$ particles and free nucleons. The SFHo EOS includes the nuclei ${ }^{2} \mathrm{H},{ }^{3} \mathrm{H},{ }^{3} \mathrm{H},{ }^{3} \mathrm{He},{ }^{4} \mathrm{Li}, \alpha$ particles, and heavy nuclei. Based on the change of the mass fractions of nuclei across the shock, the nuclear dissociation parameter is calculated using formula (B7) derived in Appendix B. The binding energies of the light nuclei are taken from the Audi, Wapstra \& Thibault (2003) data base, while that of heavy nuclei are assumed to be equal to that of iron nuclei, i.e. $8.8 \mathrm{MeV}$ per nucleon. For calculating the dissociation energy at the shock, this is a reasonable assumption as the binding energies of heavy nuclei in the iron core and $\mathrm{Si} / \mathrm{O}$ shells differ by at most $\sim 10$ per cent.

The qualitative behaviours of $\varepsilon$ and $M_{1}$ depend on the overall dynamics of each model. In this respect, all the models considered here can be categorized into three groups: (i) non-exploding models, in which the shock wave gradually decreases with time without exhibiting strong radial oscillations (group I); (ii) exploding models, in which the shock gradually expands without strong oscillations (group II); and (iii) models, in which the shock wave exhibits strong oscillations before either transitioning to explosion or failing to explode (group III). In the following, it is described these three different model groups separately.

The top panel of Fig. 11 shows the shock radius (solid black line) and the dissociation parameter $\varepsilon$ (solid red line) as a function of post-bounce time for model $s 15 h 1.22$. This is a non-exploding model, in which the shock gradually recedes without exhibiting strong radial oscillations, i.e. this model belongs to group I. After the initial period of $\sim 50 \mathrm{~ms}$, during which shock undergoes rapid expansion, the shock stalls until $t-t_{\mathrm{b}} \sim 100 \mathrm{~ms}$, after which $R_{\text {shock }}$ starts receding monotonically. The qualitative behaviour of $\varepsilon$ is similar to that of $R_{\text {shock }}$ : following the initial period of increase and subsequent stagnation, $\varepsilon$ gradually decreases with time. The dissociation parameters $\varepsilon$ falls below, e.g. $\varepsilon=0.2$ when $R_{\text {shock }} \lesssim 55 \mathrm{~km}$. Other models of group I exhibit a similar behaviour.

The centre panel of Fig. 11 shows the shock radius (dashed black line) and the dissociation parameter $\varepsilon$ (solid red line) as a function of post-bounce time for model $s 15 h 1.23$. This is an exploding model, in which the shock gradually expands without exhibiting strong radial oscillations, i.e. it belongs to group II. In this model, the stalled shock phase lasts until $t-t_{\mathrm{b}} \sim 200 \mathrm{~ms}$, after which $R_{\text {shock }}$ slowly increases. In this phase, $R_{\text {shock }}$ exhibits only weak oscillations with a relative amplitude of a few per cent. At $t-t_{\mathrm{b}} \sim 500 \mathrm{~ms}$, the shock starts rapidly expanding and the model quickly transitions towards explosion. In the early $t-t_{\mathrm{b}} \lesssim 500 \mathrm{~ms}$ after bounce, the dissociation parameter stays above 0.2 and oscillates around the value of $\sim 0.5$. However, it rapidly decreases during the explosion phase, once the shock radius becomes $\gtrsim 800 \mathrm{~km}$. Other models of group II exhibit a similar behaviour.

It is illuminating to analyse $\varepsilon$ as a function of shock radius, a plot of which is shown in Fig. 12 for all of the models in groups I and II. Each line in this plot corresponds to one model and the colour of a point on this line reflects that the time after bounce: the blue end of each line corresponds to $t-t_{\mathrm{b}}=10 \mathrm{~ms}$, while the red part corresponds to the end of the simulations $\left(t-t_{\mathrm{b}} \sim 1 \mathrm{~s}\right)$. In all non-exploding models (group I), $\varepsilon$ scales as $\propto R_{\text {shock }}$, with the
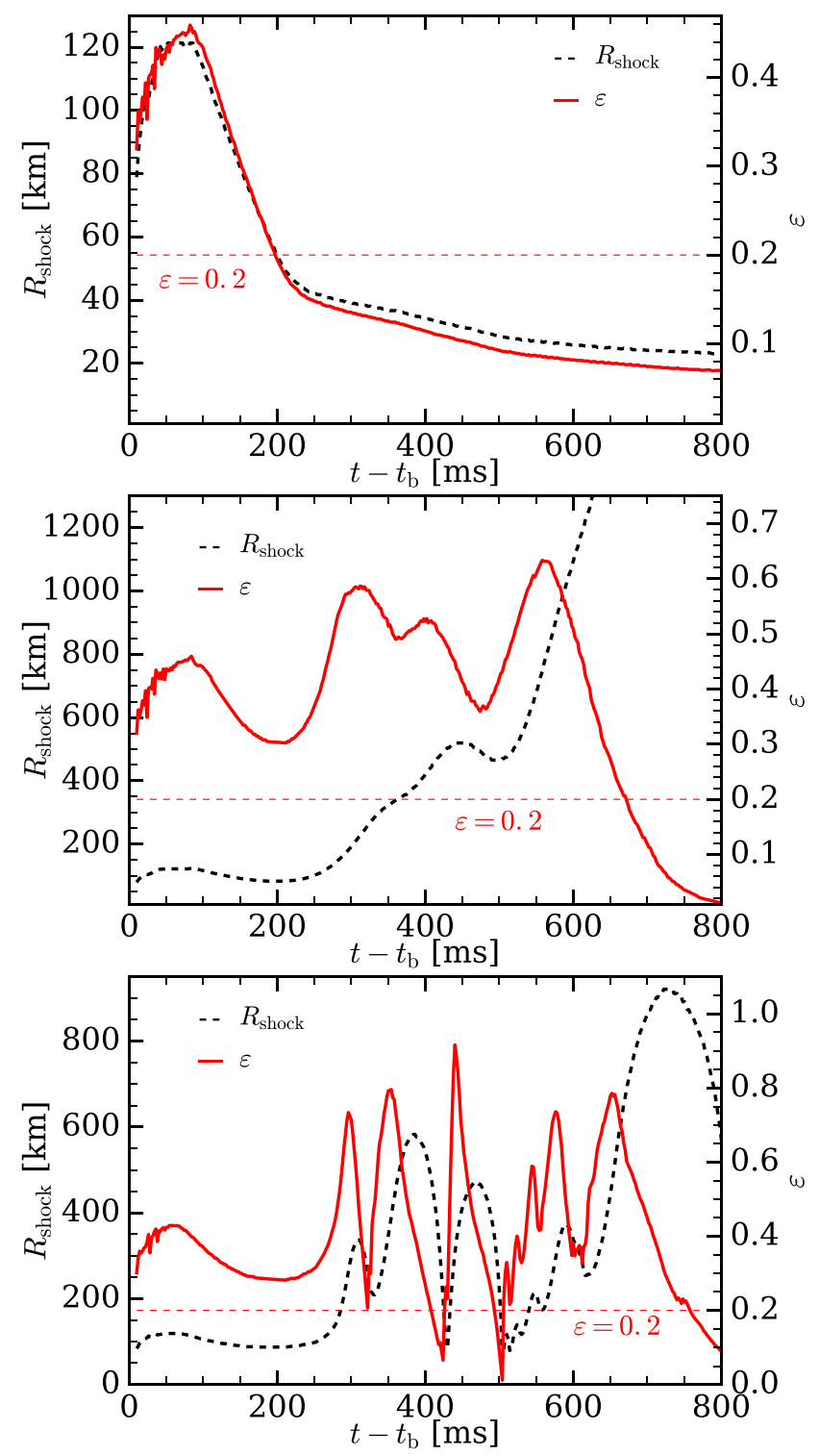

Figure 11. Top panel: time evolution of the shock radius (dashed black line) and the nuclear dissociation parameter (solid red line) for non-exploding model $s 15$ with heating factor $h=1.2$ (i.e. a group I model). For reference, the horizontal red dashed line shows the $\varepsilon=0.2$ line. Centre panel: the same as in top panel but for exploding model $s 15$ with heating factor $h=1.23$ (i.e. a group II model). Bottom panel: the same as in top panel but for model $s 25$ with heating factor $h=1.18$ that undergoes strong shock oscillations (i.e. a group III model). Such oscillations, which lead to large variations in the values of $\varepsilon$, are artificially strong in $1 \mathrm{D}$ simulations compared to 3D models. Hence, such models are not included in the analysis.

proportionality depending on mass:

$\varepsilon \sim \frac{2}{3} M_{1.3}^{-1}\left(\frac{R_{\text {shock }}}{150 \mathrm{~km}}\right)$.

This relation is qualitatively similar to equation (4) predicted by Fernández \& Thompson (2009a). However, as can be seen in Fig. 12, the $\varepsilon \propto R_{\text {shock }}$ scaling becomes invalid as soon as shock becomes larger than $\sim 175 \mathrm{~km}$, which occurs in exploding models. In this regime, $\varepsilon$ stops growing with $R_{\text {shock }}$ and saturates to $\sim 0.5$ for most models. 


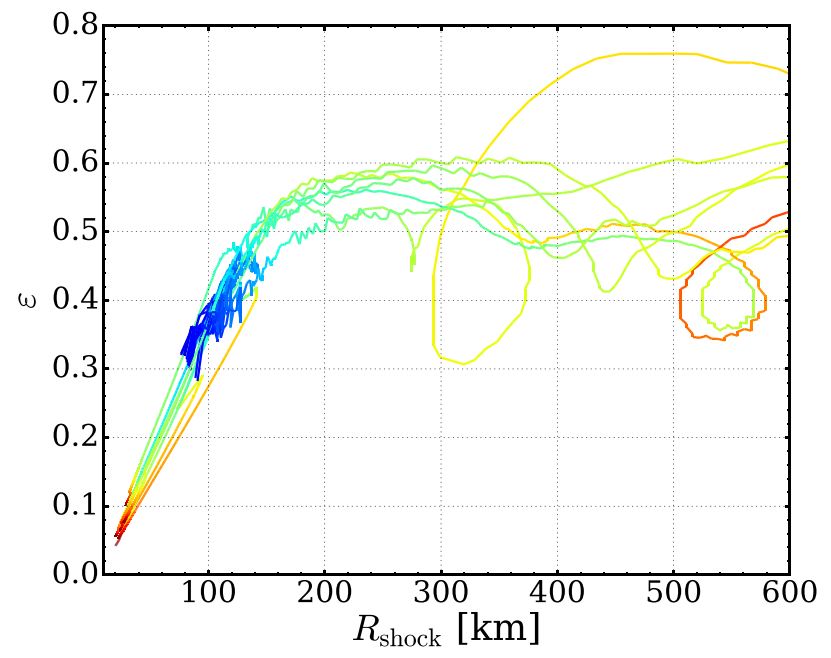

Figure 12. The nuclear dissociation parameter $\varepsilon$ as a function of the shock radius for non-exploding (group I) and exploding (group II) models. Each line represents a specific model and the colour of each line indicates the time: the blue end of the line corresponds to $10 \mathrm{~ms}$ after bounce, while the red end corresponds to late post-bounce time $\left(t-t_{\mathrm{b}} \sim 1 \mathrm{~s}\right)$. For shock radii $R_{\text {shock }} \lesssim 175 \mathrm{~km}, \varepsilon$ scales as $\propto R_{\text {shock }}$, while for large shock radii, the growth of $\varepsilon$ saturates and remains $\sim 0.5$ until $R_{\text {shock }} \lesssim 600 \mathrm{~km}$.

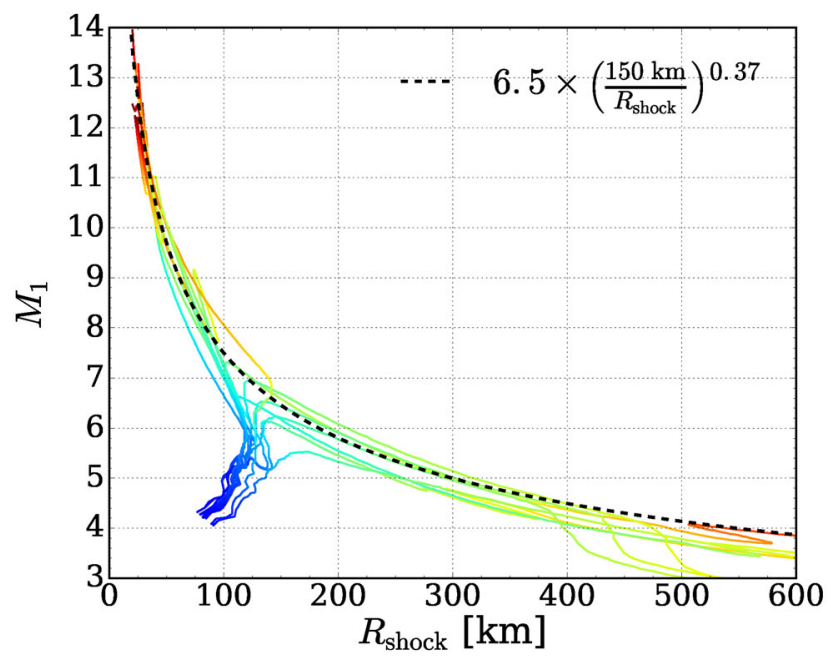

Figure 13. Mach number as a function of the shock radius. The colour of each line indicates the corresponding post-bounce time at which this value of the Mach number is extracted. The blue end of the lines corresponds to early post-bounce time of $t-t_{\mathrm{b}}=10 \mathrm{~ms}$, while the red region corresponds to late post-bounce time $\left(t-t_{\mathrm{b}} \sim 1 \mathrm{~s}\right)$. The dashed black line represents fitting function (52) that yields the values of the pre-shock Mach number as a function of the shock radius $R_{\text {shock }}$ in the stalled shock phase.

Fig. 13 shows pre-shock Mach number $M_{1}$ as a function of shock radius for all of the models in groups I and II. As in Fig. 12, each line represents a single model and the colour of each point on each line represents the post-bounce time. Except the immediate post-bounce time ( $t-t_{\mathrm{b}} \sim 10-20 \mathrm{~ms}$ ), $M_{1}$ depends on $R_{\text {shock }}$ as

$M_{1} \sim 6.5 \times\left(\frac{150 \mathrm{~km}}{R_{\text {shock }}}\right)^{0.37}$.

This relation is only approximate and the spread of the values of $M_{1}$ at a given $R_{\text {shock }}$ is caused by the fact that different models have

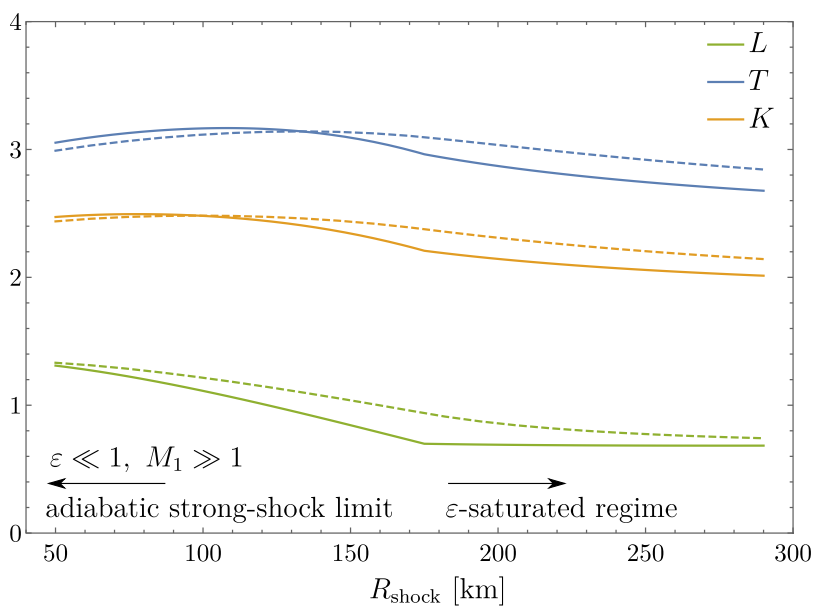

Figure 14. Longitudinal $L$, transverse $T$, and total $K$ kinetic energy amplification factors as a function of the shock radius. The dashed lines represent the amplification of the integration kinetic energy in the post-shock region (cf. equation 53).

somewhat different thermodynamic conditions (e.g. temperature), which leads to different values of the speed of sound, which, in turn, affects the Mach number.

Finally, the bottom panel of Fig. 11 shows the shock radius (solid black line) and the nuclear dissociation parameter $\varepsilon$ (solid red line) as a function of time for models $s 25 h 1.18$. This model exhibits strong radial shock oscillations from $\sim 200$ till $\sim 800 \mathrm{~ms}$ after bounce. During this time, $\varepsilon$ also undergoes strong oscillations with the same frequency as the shock radius. The oscillations in the two quantities are somewhat out of phase. When the increase of $R_{\text {shock }}$ is decelerating, $\varepsilon$ starts decreasing fast, reaching its local minimum just before $R_{\text {shock }}$ does. It starts increasing when the shock radius is approaching its local minimum. At its minimum, $\varepsilon$ can become as small as 0.1 for a brief period of time. The frequency of these oscillations is comparable to the frequencies of the in-falling perturbations. For this reason, the decomposition of the flow into stationary and fluctuating parts that the linear formalism employs is unlikely to be applicable to such models (cf. Section 2.2). On the other hand, such oscillations are artificially strong in 1D models. Full 3D simulations are unlikely to exhibit strong oscillations, at least not in the angle-averaged shock radius. However, in the presence of strong SASI oscillations, the shock radius may oscillate along radial directions. In these situations, the values of $\varepsilon$ are likely exhibit similar oscillations as in models in group III.

\subsection{Amplification of turbulent kinetic energy as a function of the shock radius}

In addition to analysing the amplification of turbulent kinetic energy across the shock as a function of parameters $\varepsilon$ and $M_{1}$, as was done in Section 4 , one can get additional insight by looking at it as a function of the shock radius $R_{\text {shock }}$. To this end, equations (51) and (52) allow us to express the nuclear dissociation degree $\varepsilon$ and shock strength $M_{1}$ as functions of the shock radius, $R_{\text {shock}}$. These expressions are employed to compute $L, T$, and $K$ as a function of $R_{\text {shock }}$ in Fig. 14. Each component of the turbulent kinetic energy appears to depend rather weakly on $R_{\text {shock }}$. The transverse component increases by a factor of $\sim 3$, while the longitudinal component experiences no significant amplification. The total turbulent kinetic energy amplifies by a factor of $\sim 2$. As dictated by computations in 
Fig. 12, there exit two distinguished regions, the zone where $\varepsilon$ is linearly proportional to the shock position $\left(R_{\text {shock }} \leq 175 \mathrm{~km}\right)$ and the region where nuclear dissociation is saturated. For small radius, the strong shock adiabatic limit applies, as $M_{1}$ grows proportional to $R_{\text {shock }}^{-0.37}$ and $\varepsilon$ approaches to zero.

The dashed lines in Fig. 14 represent the amplification of the integrated kinetic energy in the region of space confined between the shock and the centre through

$$
\left(\begin{array}{c}
\bar{L} \\
\bar{T} \\
\bar{K}
\end{array}\right)=\frac{3}{R_{\text {shock }}^{3}} \int_{0}^{R_{\text {shock }}}\left(\begin{array}{c}
L(r) \\
T(r) \\
K(r)
\end{array}\right) r^{2} \mathrm{~d} r,
$$

provided that the characteristic time of post-shock turbulent structures evolution due to viscous-diffusive effects is much longer than shock time passage through the matter to the distance $R_{\text {shock }}$.

\section{DISCUSSION: IMPACT ON THE CCSN EXPLOSION MECHANISM}

Generally speaking, the pre-shock perturbations in CCSNe consist of different physical modes, including acoustic and entropy waves in addition to the vorticity modes considered in this work. Without including all of these modes, one cannot obtain a rigorous estimate of the impact of perturbations on the explosion condition. However, as there are not theoretical models capable of describing how these perturbations are correlated in pre-collapse conditions, to the best of the authors knowledge, the effect of vorticity modes alone has been preferred to be studied in this work. The effect of other modes will be given in a future work.

The impact of the perturbations on the explosion condition can be analysed using the concept of the critical neutrino luminosity, i.e. the minimum neutrino luminosity that is necessary in order to produce an explosion for a given stellar model (Burrows \& Goshy 1993). The turbulence behind the supernova shock reduces the critical luminosity, an analytical estimate of which was obtained by Müller \& Janka (2015):

$L_{\text {crit }} \propto(\dot{M} M)^{3 / 5} r_{\text {gain }}^{-2 / 5}\left(1+\frac{4}{3}\left\langle\delta M_{2}^{2}\right\rangle\right)^{-3 / 5}$,

where $\delta M_{2}$ is the turbulent Mach number in the gain region. It is composed of two contributions, one coming from neutrino-driven convection and/or SASI, another stemming from the perturbations crossing the shock. Müller \& Janka (2015) argue that the impact of the density perturbations generated by the advection of vorticity waves plays the dominant role in driving buoyancy-driven turbulence in the post-shock region. The density perturbations become buoyant and drive additional turbulence in the post-shock region. Using a simplified model, the resulting reduction in the critical luminosity was recently estimated by Müller et al. (2016):

$\frac{\Delta L_{\text {crit }}}{L_{\text {crit }}} \simeq-\frac{0.15 \pi}{l \eta_{\text {acc }} \eta_{\text {heat }}} \sqrt{\left\langle\delta M_{0}^{2}\right\rangle}$,

where $\delta M_{0}$ is the turbulent Mach number in the convective nuclear burning shell prior to collapse, $l$ is the angular wavenumber of the dominant perturbation, $\eta_{\text {heat }}$ and $\eta_{\text {acc }}$ are the efficiencies of neutrino heating and accretion.

Estimate (55) is based on a number of assumptions and approximations. In particular, equation (55) is derived under the assumption that the advection of convective perturbations from $\mathrm{Si} / \mathrm{O}$ shells towards the shock generates density perturbations of order

$\sqrt{\left\langle\bar{\rho}_{2}^{2}\right\rangle} \sim \sqrt{\left\langle\delta M_{0}^{2}\right\rangle}$ behind the shock. 3D neutrino-hydrodynamics simulations of Müller et al. (2017) confirm that this scaling is roughly valid for their $18 \mathrm{M}_{\odot}$ model. However, this estimate does not include the density fluctuations associated with entropy perturbations generated in the post-shock region by the interaction of the shock with vorticity waves. Below, it is presented an estimate the impact of these perturbations on the critical luminosity, followed by a discussion of the critical assumptions that are employed in the analysis.

\subsection{Density perturbations in the post-shock region}

According to the linearized Rankine-Hugoniot equations, equations (17a)-(17c), the corrugated shock front induces density perturbations in the post-shock gas. Such perturbations are of entropic $\left(\hat{\rho}_{\mathrm{e}}\right)$ and acoustic $\left(\hat{\rho}_{\mathrm{a}}\right)$ nature, with the former remaining frozen to the fluid particles in the absence of diffusive effects. For isotropic field of incoming vorticity perturbations, the average of the squared density changes in the post-shock region can be written as

$\left\langle\bar{\rho}^{2}\right\rangle=D \int_{0}^{\infty} \hat{u}_{1}^{2}(k) k^{2} \mathrm{~d} k$,

with the dimensional pre-spectrum coefficient $D$, split into entropic $D_{\text {e }}$ and acoustic $D_{\text {a }}$ contributions, being computed as

$$
\begin{aligned}
D= & D_{\mathrm{e}}+D_{\mathrm{a}}=(\mathcal{D}-1)^{2} \int_{0}^{1}\left(\mathcal{P}_{\mathrm{li}}^{2}+\mathcal{P}_{\mathrm{li}}^{2}\right) P(\zeta) \mathrm{d} \zeta \\
& +(\mathcal{D}-1)^{2} \int_{1}^{\infty} \mathcal{P}_{\mathrm{s}}^{2} P(\zeta) \mathrm{d} \zeta+\int_{1}^{\infty} \mathcal{P}^{2} P(\zeta) \mathrm{d} \zeta
\end{aligned}
$$

The terms involving the factor $(\mathcal{D}-1)^{2}$ correspond to the entropic contribution $D_{\mathrm{e}}$, while the last term refers to the acoustic part $D_{\mathrm{a}}$.

Fig. 15 shows the function $D_{\mathrm{e}}$ and $D_{\mathrm{a}}$ versus $M_{1}$ for $\varepsilon=0,0.2$, and 0.4. Both $D_{\mathrm{e}}$ and $D_{\mathrm{a}}$ grows with $M_{1}$ and $\varepsilon$. The acoustic part $D_{\mathrm{a}}$ is at least two orders of magnitude smaller than the entropic part $D_{\mathrm{e}}$ and thus it is negligible.

In order to obtain a more intuitive insight, it is useful to express $\left\langle\bar{\rho}^{2}\right\rangle$ as a function of the pre-shock turbulent Mach number. The latter is related to the average upstream velocity perturbations as

$\left\langle\delta M_{1}^{2}\right\rangle=3\left(\frac{a_{2}}{a_{1}}\right)^{2}\left\langle\bar{u}_{1}^{2}\right\rangle=\frac{3 M_{1}^{2}}{M_{2}^{2} C_{2}^{2}}\left\langle\bar{u}_{1}^{2}\right\rangle$

that combined with equations (38) and (57) yields

$\left\langle\bar{\rho}_{2}^{2}\right\rangle=\frac{M_{2}^{2} C_{2}^{2} D}{8 \pi M_{1}^{2}}\left\langle\delta M_{1}^{2}\right\rangle=A\left\langle\delta M_{1}^{2}\right\rangle$.

Fig. 16 shows the function that correlates the upstream turbulent Mach number with the downstream average density perturbations, $A=\left\langle\bar{\rho}_{2}^{2}\right\rangle /\left\langle\delta M_{1}^{2}\right\rangle$, as a function of the shock strength $M_{1}$ for $\varepsilon=0$, 0.2 , and 0.4 . For typical values of these parameters $(0.2 \lesssim \varepsilon \lesssim$ 0.4 and $\left.M_{1} \gtrsim 5\right)$, the ratio $\left\langle\bar{\rho}_{2}^{2}\right\rangle /\left\langle\delta M_{1}^{2}\right\rangle$ ranges from $\simeq 0.1$ to $\simeq 0.2$. Accordingly,

$\sqrt{\left\langle\bar{\rho}_{2}^{2}\right\rangle} \simeq(0.32-0.45) \times \sqrt{\left\langle\delta M_{1}^{2}\right\rangle}$.

The turbulent Mach number $\sqrt{\left\langle\delta M_{1}^{2}\right\rangle}$ immediately above the shock is readily related to that in the pre-collapse convective shells. During collapse, the Mach number of vorticity waves grows as $\propto r^{(3 \gamma-7) / 4}$ in the absence of dissipative effects (Kovalenko \& Eremin 1998; Lai \& Goldreich 2000). If the convective shell falls from a radius of $\sim 1500$ to $\sim 200 \mathrm{~km}$, the turbulent Mach number should increase by a factor of $\sim 4.53$. Applying this to scaling (61) gives

$\sqrt{\left\langle\bar{\rho}_{2}^{2}\right\rangle} \simeq(1.45-2.04) \times \sqrt{\left\langle\delta M_{0}^{2}\right\rangle}$. 

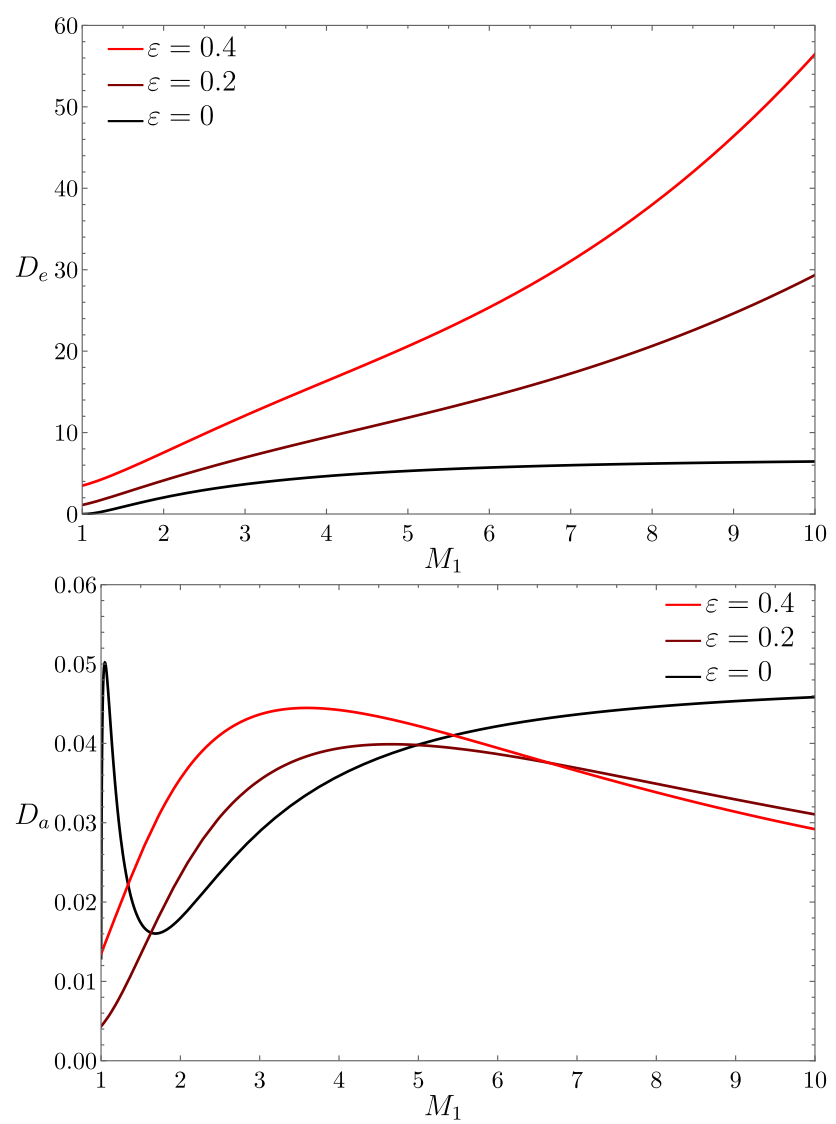

Figure 15. Pre-spectrum function $D$ associated with density perturbations as a function of the shock intensity $M_{1}$ for dissociation energy parameters $\varepsilon=0,0.2$, and 0.4 . The upper panel shows the contribution of entropicrotational perturbations $D_{\mathrm{e}}$, and the lower panel displays the acoustic contribution $D_{\mathrm{a}}$.

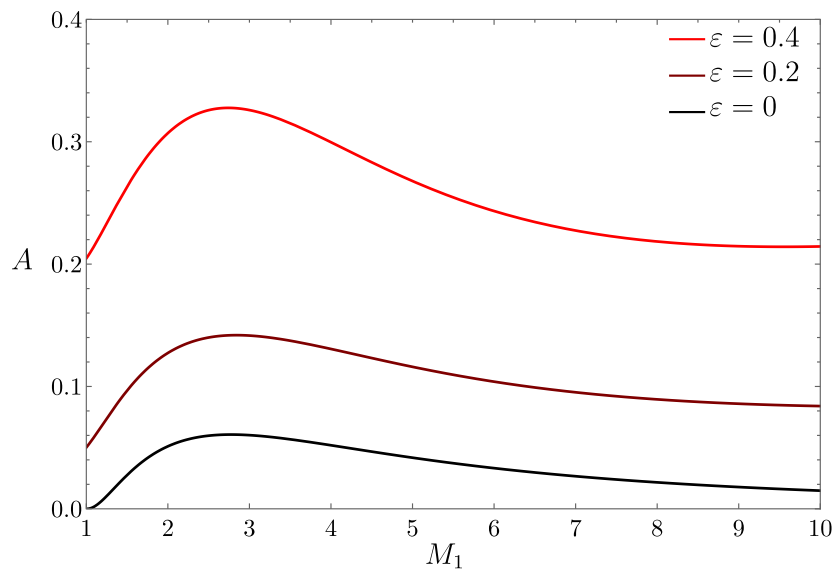

Figure 16. Correlation function of downstream average density perturbations and upstream turbulent Mach number $A=\left\langle\bar{\rho}_{2}^{2}\right\rangle /\left\langle\delta M_{1}^{2}\right\rangle$ as a function of the shock intensity $M_{1}$ for dissociation energy parameters $\varepsilon=0,0.2$, and 0.4 . These density fluctuations are predominantly caused by entropy perturbations, with the contribution of acoustic waves being two orders of magnitude smaller. The density perturbations become buoyant and generate additional convection in the post-shock region, creating more favourable condition for shock revival.
Thus, the density perturbations predicted by this relation is larger by a factor of $\simeq 1.45-2.04$ than that generated by the advection of the vorticity waves given by equation (56). Below, it is investigated if these perturbations contribute to the turbulence in the gain region.

\subsection{Generation of turbulence from density perturbations}

When density perturbations are immersed in a gravitational field, buoyancy effects may play a significant role in contributing to the turbulent kinetic energy. The kinetic energy production or consumption can be scaled with $\langle\bar{\rho} \bar{u}\rangle g / a_{2}$ (see e.g. chapter 8.2 of Holton \& Hakim 2012), with $\bar{u}$ being the velocity component parallel to the gravity field $g$, which in the case considered coincides with the direction of the mean flow. However, as gas dynamics equations in equation (15) do not take into account the effect of body forces, the model shown in this paper can only predict the initial state of density and velocity perturbations generated by the shock. Thus, the shock-generated density-velocity correlation will seed an initial entrainment of kinetic energy akin to buoyancy perturbations, which may later be self-amplified if local conditions are suitable for that to happen. As background temperature profiles are expected to decay with the radius (the shock propagation direction coordinate), the velocity-density correlation involved in buoyancy-driven kinetic energy is thought to contribute positively to the total turbulent kinetic energy downstream. Nonetheless, as the shock passage time frame is very short, the critical conditions are expected to be dominated by the post-shock state rather than time-dependent turbulence-evolving effects.

With these considerations in mind, the correlation of velocity and density disturbances is defined as

$\langle\bar{\rho} \bar{u}\rangle=B \int_{0}^{\infty} \hat{u}_{1}^{2}(k) k^{2} \mathrm{~d} k$,

where $B$ is a dimensionless pre-spectrum factor,

$$
\begin{aligned}
B= & B_{\text {er }}+B_{\mathrm{a}}=(\mathcal{D}-1) \int_{0}^{1}\left(\mathcal{U}_{\mathrm{lr}}^{\mathrm{r}} \mathcal{P}_{\mathrm{lr}}+\mathcal{U}_{\mathrm{li}}^{\mathrm{r}} \mathcal{P}_{l i}\right) P(\zeta) \mathrm{d} \zeta \\
& +(\mathcal{D}-1) \int_{1}^{\infty} \mathcal{U}_{\mathrm{s}}^{\mathrm{r}} \mathcal{P}_{\mathrm{s}} P(\zeta) \mathrm{d} \zeta+\int_{1}^{\infty} \mathcal{U}^{\mathrm{a}} \mathcal{P} P(\zeta) \mathrm{d} \zeta .
\end{aligned}
$$

The entropic-rotational part are the terms proportional to the factor $\mathcal{D}-1$, while the last integral represents the acoustic contribution. For negative values of $\langle\bar{\rho} \bar{u}\rangle$ (i.e. positive velocity-temperature correlation), the density perturbation contributes constructively to the post-shock turbulent kinetic energy. The contrary applies for $\langle\bar{\rho} \bar{u}\rangle>0$.

Fig. 17 shows the correlation function $B$ as a function of the shock Mach number for $\varepsilon=0,0.2$, and 0.4. Similarly to $D$, the acoustic contribution to $B$ is found to be negligible. The buoyancy perturbations are negative, meaning that the density perturbations will increase the value of the final turbulent kinetic energy.

In the light of this finding, density fluctuations (equation 62) from entropy waves are substituted into the expression for the reduction of the critical luminosity (equation 55) and obtain

$\frac{\Delta L_{\text {crit }}}{L_{\text {crit }}} \simeq-(1.45-2.04) \times \frac{0.15 \pi}{l \eta_{\text {acc }} \eta_{\text {heat }}} \sqrt{\left\langle\delta M_{0}^{2}\right\rangle}$.

For typical values of $\eta_{\text {acc }}=2, \eta_{\text {heat }}=0.1, \sqrt{\left\langle\delta M_{0}^{2}\right\rangle} \sim 0.1$, and $l=2$, it is obtained $17-24$ per cent reduction in the critical luminosity. This roughly agrees with the results of 3D simulations (Müller et al. 2017). 


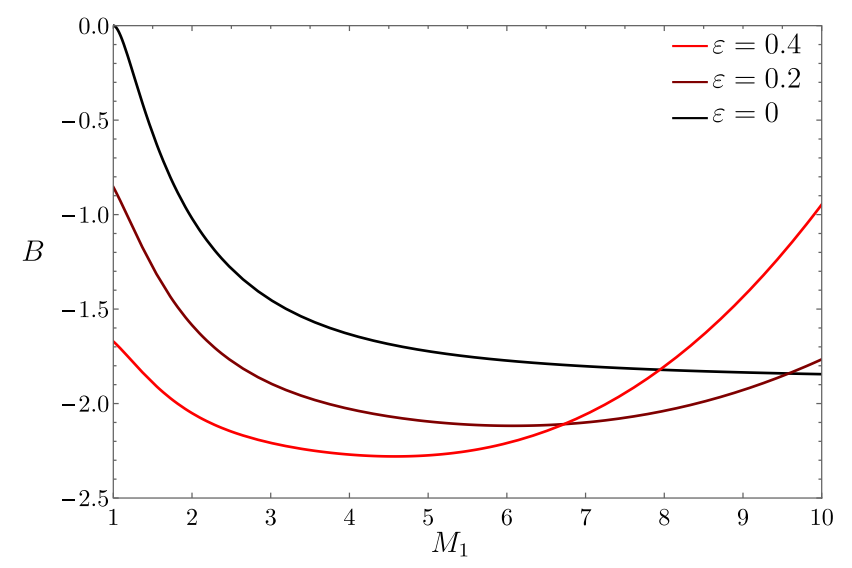

Figure 17. Density-velocity correlation function $B$ as a function of the shock intensity $M_{1}$ for dissociation energy parameters $\varepsilon=0,0.2$, and 0.4 .

\subsection{Impact of acoustic waves and direct injection of kinetic energy}

In addition to the impact of entropy perturbations on the explosion condition of CCSNe, one can in principle study the role of other effects such as the acoustic waves generated by the interaction of the shock with vorticity waves and the direct injection of the kinetic energy by vorticity waves to the post-shock region. The reduction of the critical luminosity due to the latter was estimated by Abdikamalov et al. (2016):

$\frac{\Delta L_{\text {crit }}}{L_{\text {crit }}} \sim 0.6\left\langle\delta M_{1}^{2}\right\rangle$

For the same parameters used for estimate (65), equation (66) yields $\sim 12$ per cent reduction in the critical luminosity. This is smaller than that due to the entropy perturbations calculated above. Hence, the direct injection of turbulent kinetic energy of vorticity waves is expected to play a smaller effect, in agreement with the estimate of Müller \& Janka (2015).

As seen above in Sections 4.1 and 6.1, the acoustic waves have negligibly small contribution to the perturbations of velocity and density compared to the contributions of the vorticity and entropy modes in the post-shock. For this reason, the acoustic waves in the post-shock region are expected to have negligibly small effect on the explosion condition of CCSNe (see also the discussion in Müller et al. 2016).

\subsection{Assessment of underlying assumptions}

Estimates (65) and (66) are based on a number of assumptions and approximations. As discussed above, equation (66) measures the impact of the direct injection of non-radial motion into the gain region by vorticity waves, which is then superposed on top of the neutrino-driven turbulent convection that is already present in the gain region by the time the vorticity waves cross the shock. This procedure implicitly assumes that the kinetic energy of vorticity waves does not change after shock crossing, which is consistent with the requirement of exact balance between turbulent dissipation and buoyant driving. Currently, it is unclear if such a balance is maintained for vorticity waves right after crossing the shock. Moreover, the accuracy is the simple superposition used by Abdikamalov et al. (2016) has not yet been verified.

Equation (65) measures the impact of the buoyancy-driven turbulent convection triggered by the entropy waves that are generated by the perturbed shock. This is based on an estimate of the turbulent kinetic energy in the gain region assuming exact balance between buoyant driving of turbulence and turbulent dissipation. This neglects possible change of size of the gain region due to heat dissipation in the gain region (Mabanta \& Murphy 2017). Moreover, the turbulence generated by upstream perturbations and the neutrino-driven turbulence that develops prior to the arrival of upstream perturbations are added together using a simple interpolation (cf. equation 46 of Müller et al. 2016). Finally, it is assumed an isotropic distribution of vorticity perturbations in the upstream flow. The validity of all of these approximations and assumptions need to be verified using accurate $3 \mathrm{D}$ neutrino-hydrodynamics simulations.

\section{CONCLUSIONS}

The shock-turbulence interplay plays a key role in facilitating CCSN explosions. In this paper, we studied how vorticity waves from nuclear shell burning affect the shock dynamics once they encounter in the aftermath of stellar core collapse. Our study accounts for the interaction of the shock with intermediate vortical scales, i.e. those whose characteristic length is sufficiently small for the shock to be considered a planar front, yet sufficiently large for the shock to be a seen as a discontinuity front. The mathematical formalism is based on the solution of the linearized hydrodynamics equations in the post-shock region (Wouchuk et al. 2009; Huete et al. 2017), which captures the full time evolution of shock-vorticity system in the linear order. In particular, this allowed us to take into account the perturbation of the nuclear dissociation itself, which was not included previously (Abdikamalov et al. 2016). We demonstrated that this effect plays an important role in shock-turbulence interaction.

When a vorticity wave encounters a shock, the shock deforms and generates a post-shock field of entropy and acoustic waves. We have analysed the properties of these fluctuations for a wide range of the parameters of the incoming vorticity waves and mean flow (Sections 3 and 4). One expected finding is that kinetic energy of solenoidal velocity perturbations is amplified as a result of the shock interaction. We have also found that, within the limits of validity of the model, density perturbations in the post-shock region are dominantly of entropic nature, while the contribution of the acoustic waves is smaller by two orders of magnitude. The contribution of acoustic waves to the non-radial kinetic energy in the post-shock region is smaller than that of rotational waves by a similar factor.

Using an approximate model for the interaction of the perturbations with the post-shock flow and a concept of critical neutrino luminosity (Burrows \& Goshy 1993), we estimate the effect of the perturbations on the explosion condition. In particular, we show that the entropy perturbations in the post-shock region are the largest factor in generating turbulence in the post-shock flow due to work by buoyancy forces (Section 6). For typical program parameters, the amplitude of density perturbations is about 1.45-2.04 times the turbulent Mach number in the $\mathrm{Si} / \mathrm{O}$ shell. We show that, for typical problem parameters, this leads to $\sim 17-24$ percent reduction in the critical luminosity for producing explosion (cf. Section 6). This approximately agrees with the results of recent 3D neutrinohydrodynamics simulations (Müller et al. 2017). On the other hand, the direct injection of non-radial motion into the post-shock region by vorticity waves reduces the critical luminosity by $\sim 12$ per cent for typical problem parameters (cf. Section 6.3), which is smaller than the impact of the buoyant entropy perturbations. Finally, due to their small kinetic energy, the acoustic waves generated by the perturbed shock (cf. Section 6.3) have negligibly small effect on the explosion condition. 
The estimate of the reduction of the critical luminosity is based on a number of assumptions and approximations (cf. Section 6.4). In particular, the turbulence driven by upstream perturbations is approximately added on top of the neutrino-driven turbulence that already exists by the time the perturbations reach the shock. The validity of such an approach has yet to be rigorously verified using accurate 3D neutrino-hydrodynamics simulations. Moreover, the interaction of pre-collapse perturbations with the hydrodynamic instabilities in the post-shock region has to be treated in a more rigorous way (e.g. by extending the ideas of Takahashi et al. 2016 and Mabanta \& Murphy 2017). Finally, one has to study the effect of other perturbation modes such as acoustic waves that originate from the accretion of convective shells. This will be the subject of future studies.

\section{ACKNOWLEDGEMENTS}

We thank B. Müller for carefully reading the manuscript and for many valuable comments that significantly improved the paper. We also thank T. Foglizzo, M. Hempel, and A. L. Velikovich for useful discussions. This work is supported by the Ministry of Science, MEC (ENE2015-65852-C2-1-R) and Fundación Iberdrola España (BINV-ua37crdy), Spain (for CH), by ORAU grant at Nazarbayev University (for EA), by Max-Planck/Princeton Center (MPPC) for Plasma Physics (NSF PHY-1144374), and a Schmidt Fellowship (for DR). The computations were performed at the NULITS Linux cluster at Nazarbayev University. We thank S. Bubin for his contribution to set up the cluster.

\section{REFERENCES}

Abdikamalov E. et al., 2015, ApJ, 808, 70

Abdikamalov E., Zhaksylykov A., Radice D., Berdibek S., 2016, MNRAS, 461,3864

Adams S. M., Kochanek C. S., Gerke J. R., Stanek K. Z., 2017, MNRAS, 469,1445

Audi G., Wapstra A. H., Thibault C., 2003, Nucl. Phys. A, 729, 337

Baade W., Zwicky F., 1934, Proc. Natl. Acad. Sci. USA, 20, 254

Batchelor G. K., 1953, The Theory of Homogeneous Turbulence. Cambridge Univ. Press, Cambridge

Bethe H. A., 1990, Rev. Modern Phys., 62, 801

Blondin J. M., Mezzacappa A., DeMarino C., 2003, ApJ, 584, 971

Bruenn S. W. et al., 2016, ApJ, 818, 123

Burrows A., 2013, Rev. Modern Phys., 85, 245

Burrows A., Goshy J., 1993, ApJ, 416, L75

Burrows A., Hayes J., Fryxell B. A., 1995, ApJ, 450, 830

Burrows A., Dessart L., Livne E., Ott C. D., Murphy J., 2007, ApJ, 664, 416

Burrows A., Vartanyan D., Dolence J. D., Skinner M. A., Radice D., 2016, Space Sci. Rev., preprint (arXiv:1611.05859)

Cardall C. Y., Budiardja R. D., 2015, ApJ, 813, L6

Chu B. T., Kovasznay L. S. G., 1957, J. Fluids Mech., 3, 494

Cobos-Campos F., Wouchuk J. G., 2014, Phys. Rev. E, 90, 053007

Collins C., Müller B., Heger A., 2018, MNRAS, 473, 1695

Couch S. M., Ott C. D., 2013, ApJ, 778, L7

Couch S. M., Ott C. D., 2015, ApJ, 799, 5

Couch S. M., Chatzopoulos E., Arnett W. D., Timmes F. X., 2015, ApJ, 808, L21

Dolence J. C., Burrows A., Murphy J. W., Nordhaus J., 2013, ApJ, 765, 110

Fernández R., 2015, MNRAS, 452, 2071

Fernandez R., Thompson C., 2009a, ApJ, 697, 1827

Fernandez R., Thompson C., 2009b, ApJ, 703, 1464

Fernández R., Müller B., Foglizzo T., Janka H.-T., 2014, MNRAS, 440, 2763

Foglizzo T., 2009, ApJ, 694, 820

Foglizzo T., Tagger M., 2000, A\&A, 363, 174
Foglizzo T., Scheck L., Janka H.-T., 2006, ApJ, 652, 1436

Foglizzo T. et al., 2015, Publ. Astron. Soc. Aust., 32, e009

Fraley G., 1986, Phys. Fluids, 29, 376

Hanke F., Marek A., Müller B., Janka H.-T., 2012, ApJ, 755, 138

Hanke F., Müller B., Wongwathanarat A., Marek A., Janka H.-T., 2013, ApJ, 770,66

Herant M., 1995, Phys. Rep., 256, 117

Holton J. R., Hakim G. J., 2012, An Introduction to Dynamic Meteorology, 5 th edn. Academic Press, Boston, MA

Huete C., Sánchez A. L., Williams F. A., 2013, Phys. Fluids, 25, 076105

Huete C., Jin. T., Martinez-Ruiz D., Luo K., 2017, Phys. Rev. E, 96, 053104

Jackson T. L., Hussaini M. Y., Ribner H. S., 1993, Phys. Fluids A: Fluid Dyn., 5, 745

Janka H.-T., Mueller E., 1996, A\&A, 306, 167

Janka H., -T., Hanke F., Hüdepohl L., Marek A., Müller B., Obergaulinger M., 2012, Progress Theor. Exp. Phys., 2012, 01A309

Kashiyama K., Quataert E., 2015, MNRAS, 451, 2656

Kovalenko I. G., Eremin M. A., 1998, MNRAS, 298, 861

Kovasznay L. S. G., 1953, J. Aeronautical Sci., 20, 657

Lai D., Goldreich P., 2000, ApJ, 535, 402

Lattimer J. M., Douglas Swesty F., 1991, Nucl. Phys. A, 535, 331

Lentz E. J. et al., 2015, ApJ, 807, L31

Lovegrove E., Woosley S. E., 2013, ApJ, 769, 109

Mabanta Q., Murphy J. W., 2017, ApJ, preprint (arXiv:1706.00072)

Mahesh K., Moin P., Lele S. K., 1996, The Interaction of a Shock Wave with a Turbulent Shear Flow. Technical Report TF-69, Thermosciences division, Department of Mechanical Engineering, Stanford University

Mao J., Ono M., Nagataki S., Hashimoto M.-a., Ito H., Matsumoto J., Dainotti M. G., Lee S.-H., 2015, ApJ, 808, 164

Melson T., Janka H.-T., Marek A., 2015, ApJ, 801, L24

Mösta P. et al., 2014, ApJ, 785, L29

Mösta P., Ott C. D., Radice D., Roberts L. F., Schnetter E., Haas R., 2015, Nature, 528, 376

Müller B., 2016, Publ. Astron. Soc. Aust., 33, e048

Müller B., Janka H.-T., 2015, MNRAS, 448, 2141

Müller B., Viallet M., Heger A., Janka H.-T., 2016, ApJ, 833, 124

Müller B., Melson T., Heger A., Janka H.-Th. 2017, MNRAS, 472, 491

Murphy J. W., Dolence J. C., Burrows A., 2013, ApJ, 771, 52

Nadezhin D. K., 1980, Ap\&SS, 69, 115

O’Connor E., Ott C. D., 2010, Classical Quantum Gravity, 27, 114103

O’Connor E., Ott C. D., 2011, ApJ, 730, 70

Ott C. D. et al., 2011, Phys. Rev. Lett., 106, 161103

Ott C. D. et al., 2013, ApJ, 768, 115

Radice D., Couch S. M., Ott C. D., 2015, Comput. Astrophys. Cosmol., 2, 7

Radice D., Ott C. D., Abdikamalov E., Couch S. M., Haas R., Schnetter E., 2016, ApJ, 820, 76

Radice D., Burrows A., Vartanyan D., Skinner M. A., Dolence J. C., 2017a, ApJ, 850, 43

Radice D., Abdikamalov E., Ott C. D., Moesta P., Couch S. M., Roberts L. F., 2017b, preprint (arXiv:1710.01282)

Ribner H. S., 1953, Convection of a Pattern of Vorticity Through a Shock Wave. Technical Report TN 2864

Roberts L. F., Ott C. D., Haas R., O’Connor E. P., Diener P., Schnetter E., 2016, ApJ, 831, 98

Steiner A. W., Hempel M., Fischer T., 2013, ApJ, 774, 17

Summa A., Janka H.-Th., Melson T., Marek A., 2018, ApJ, 852, 28

Takahashi K., Yamada S., 2014, ApJ, 794, 162

Takahashi K., Iwakami W., Yamamoto Y., Yamada S., 2016, ApJ, 831, 75

Takiwaki T., Kotake K., Suwa Y., 2014, ApJ, 786, 83

Takiwaki T., Kotake K., Suwa Y., 2016, MNRAS, 461, L112

Ugliano M., Janka H.-T., Marek A., Arcones A., 2012, ApJ, 757, 69

Velikovich A. L., Murakami M., Taylor B. D., Giuliani J. L., Zalesak S. T., Iwamoto Y., 2016, Phys. Plasmas, 23, 052706

Woosley S. E., Heger A., 2007, Phys. Rep., 442, 269

Wouchuk J. G., 2001a, Phys. Plasmas, 8, 2890

Wouchuk J. G., 2001b, Phys. Rev. E, 63, 056303

Wouchuk J. G., Huete Ruiz de Lira C., Velikovich A. L., 2009, Phys. Rev. E, 79, 066315 
Yamasaki T., Yamada S., 2006, ApJ, 650, 291

Zaidel P. M., 1960, J. Appl. Math. Mech., 24, 316

\section{APPENDIX A: LAPLACE TRANSFORM FOR THE SHOCK TEMPORAL EVOLUTION AND ASYMPTOTIC EXPRESSIONS}

The transient response of the CCSN shock wave is analysed employing a similar approach as that used in Wouchuk et al. (2009), where the transformation

$x=r \sinh \chi, \quad \tau=r \cosh \chi$

is conveniently introduced, with $\tau=0$ being the moment at which the initially planar shock front encounters the perturbed field. It is readily seen that $\chi=$ const represents a planar front moving in the burnt gas along the $x$-axis, then sweeping the burnt-gas domain from the weak discontinuity $x=0(\chi=0)$ to the detonation front $x=M_{2} \tau\left(\tanh \chi_{\mathrm{s}}=M_{2}\right)$. The sound wave equation (16) reduces to

$r \frac{\partial^{2} \bar{p}}{\partial r^{2}}+\frac{\partial \bar{p}}{\partial r}+r \bar{p}=\frac{1}{r} \frac{\partial^{2} \bar{p}}{\partial \chi^{2}}$,

while the shock boundary condition at the front becomes

$$
\begin{aligned}
\frac{\mathrm{d} \xi_{\mathrm{s}}(r)}{\mathrm{d} r}= & \frac{\sigma_{\mathrm{a}}}{\sqrt{1-M_{2}^{2}}} \bar{p}_{\mathrm{s}}(r)+\frac{\hat{u}_{1}}{\sqrt{1-M_{2}^{2}}} \cos (\zeta r), \\
\left.\frac{1}{r} \frac{\partial \bar{p}_{\mathrm{s}}}{\partial \chi}\right|_{\mathrm{s}}= & -\sigma_{\mathrm{b}} \frac{\partial \bar{p}_{\mathrm{s}}(r)}{\partial r}-\frac{M_{2}^{2}\left(C_{2}-1\right)}{\sqrt{1-M_{2}^{2}}} \xi_{\mathrm{s}}(r) \\
& +\hat{u}_{1} \zeta \frac{C_{2}-1}{C_{2}} \sin (\zeta r),
\end{aligned}
$$

where $\zeta$ is the characteristic shock oscillation frequency provided in equation (27).

The Laplace transform over the variable $r$,

$\mathscr{F}(s, \chi)=\int_{0}^{\infty} f(r, \chi) \mathrm{e}^{-s r} \mathrm{~d} r$,

is used to reduce the above shock boundary condition to an algebraical system as function of the Laplace variable $s$. The isolated boundary condition is used to compute the Laplace transform of $\left.\frac{1}{r} \frac{\partial \bar{p}_{\mathrm{s}}}{\partial \chi}\right|_{\mathrm{s}}$ as $\sqrt{s^{2}+1} \mathscr{P}_{\mathrm{s}}-\bar{p}_{\mathrm{s} 0}$, thereby giving

$$
\begin{aligned}
\mathscr{P}_{\mathrm{s}}(s)= & \frac{s\left(1+\sigma_{\mathrm{b}}\right)}{s \sqrt{s^{2}+1}+\sigma_{\mathrm{b}} s^{2}+\sigma_{\mathrm{c}}} \bar{p}_{\mathrm{s} 0} \\
& +\frac{s \sigma}{\left(s \sqrt{s^{2}+1}+\sigma_{\mathrm{b}} s^{2}+\sigma_{\mathrm{c}}\right)\left(s^{2}+\zeta^{2}\right)} \hat{u}_{1}
\end{aligned}
$$

for the Laplace transform of the pressure perturbation at the shock, with

$\sigma=\frac{C_{2}-1}{C_{2}}\left(\zeta^{2}-\frac{M_{1}^{2}}{M_{1}^{2}-1}\right)$

representing the periodic excitation amplitude as a function of $\zeta$, the characteristic oscillation frequency in the $r$-domain variable, and

$\sigma_{\mathrm{c}}=\frac{M_{2}^{2}\left(C_{2}-1\right)}{1-M_{2}^{2}} \sigma_{\mathrm{a}}$

standing for the scaled $\sigma_{\mathrm{a}}$ factor. The solution for the pressure field in equation (A2) can be expressed as a combination of the Bessel functions (Zaidel 1960),

$\bar{p}(r, \chi)=\sum_{\nu=0}^{\infty} A_{v}\left(B_{v_{1}} \mathrm{e}^{v \chi}+B_{v_{2}} \mathrm{e}^{-v \chi}\right) J_{v}(r)$, provided that the solution for Bessel function of the second type must be excluded in order to avoid a divergent behaviour when $r \rightarrow 0$. The Laplace transform of equation (A9) at the shock front yields

$\mathscr{P}_{\mathrm{s}}(s)=\sum_{\nu=0}^{\infty} N_{v} \frac{\mathrm{e}^{-v \sinh ^{-1} s}}{\sqrt{s^{2}+1}}$,

with $N_{v}=A_{v}\left(B_{v_{1}} \mathrm{e}^{v \chi_{\mathrm{s}}}+B_{v_{2}} \mathrm{e}^{-v \chi_{\mathrm{s}}}\right)$ referring to the coefficients that accompany the Bessel functions at the shock front in equation (28). Combination of equation (A10) with equation (A6) is used to provide

$N_{v}=\frac{N_{v-8} a_{4_{-}}+N_{v-6} a_{2_{-}}+N_{v-4} a_{\mathrm{s}}+N_{v-2} a_{2_{+}}}{a_{4_{+}}}$

for the even indices, with the odd values $N_{2 v+1}$ found all to be zero. The initial coefficients of the recurrence equation are

$N_{0}=\frac{1}{a_{4_{+}}}$,

$N_{2}=\frac{D_{0} a_{2_{+}}-b}{a_{4_{+}}}$

$N_{4}=\frac{D_{0} a_{\mathrm{s}}+D_{2} a_{2_{+}}}{a_{4_{+}}}$,

$N_{6}=\frac{D_{0} a_{2_{-}}+D_{2} a_{\mathrm{s}}+D_{4} a_{2_{+}}+b}{a_{4_{+}}}$,

$N_{8}=\frac{D_{0} a_{4_{-}}+D_{2} a_{2_{-}}+D_{4} a_{\mathrm{s}}+D_{6} a_{2_{+}}-1}{a_{4_{+}}}$,

with the parameters

$a_{4_{+}}=-\left(1+\sigma_{\mathrm{b}}\right), \quad a_{4_{-}}=-\left(1-\sigma_{\mathrm{b}}\right)$,

$a_{2_{+}}=2\left[2 \sigma_{\mathrm{c}}-\sigma_{\mathrm{b}}+\left(\sigma_{\mathrm{b}}+1\right)\left(2 \zeta^{2}-1\right)\right]$,

$a_{2_{-}}=2\left[2 \sigma_{\mathrm{c}}-\sigma_{\mathrm{b}}+\left(\sigma_{\mathrm{b}}-1\right)\left(2 \zeta^{2}-1\right)\right]$,

$a_{4_{-}}=2\left[\sigma_{\mathrm{b}}+2\left(2 \sigma_{\mathrm{b}}-\sigma_{\mathrm{b}}\right)\left(2 \zeta^{2}-1\right)\right]$,

$$
b=2+4\left(\sigma_{3}-\zeta^{2}\right) \text {. }
$$

The ripple amplitude is easily computed through temporal integration of equation (20) yielding

$$
\begin{aligned}
\xi_{\mathrm{s}}(r)= & \sum_{\nu=0}^{\infty} N_{\nu} \frac{r^{k+1}}{2^{k} \Gamma(2+k)} \mathcal{H}\left(\frac{1+k}{2}, \frac{3+k}{2}, 1+k,-\frac{r^{2}}{4}\right) \\
& +\frac{\hat{u}_{1}}{\zeta \sqrt{1-M_{2}^{2}}} \cos (\zeta r),
\end{aligned}
$$

with $\Gamma$ being the Euler gamma function and $\mathcal{H}$ representing the general hypergeometric function.

In the following, the expressions for the amplitudes of the periodic perturbations downstream are provided.

The long-time response of the shock pressure evolution is, as shown in equation (26),

$\bar{p}_{\mathrm{s}}(\tau \gg 1)= \begin{cases}\mathcal{P}_{\mathrm{lr}} \cos \left(\omega_{\mathrm{s}} \tau\right)+\mathcal{P}_{\mathrm{li}} \sin \left(\omega_{\mathrm{s}} \tau\right), & \zeta \leq 1, \\ \mathcal{P}_{\mathrm{s}} \cos \left(\omega_{\mathrm{s}} \tau\right), & \zeta \geq 1,\end{cases}$

whose coefficients for the long-wavelength $\left(\mathcal{P}_{\text {lr }}\right.$ and $\left.\mathcal{P}_{\text {li }}\right)$ and shortwavelength regime $\left(\mathcal{P}_{\mathrm{s}}\right)$ are

$\mathcal{P}_{\mathrm{lr}}=\frac{-\sigma\left(\sigma_{\mathrm{b}} \zeta^{2}-\sigma_{\mathrm{c}}\right)}{\zeta^{2}\left(1-\zeta^{2}\right)+\left(\sigma_{\mathrm{b}} \zeta^{2}-\sigma_{\mathrm{c}}\right)}$, 
$\mathcal{P}_{\mathrm{li}}=\frac{\sigma \zeta \sqrt{1-\zeta^{2}}}{\zeta^{2}\left(1-\zeta^{2}\right)+\left(\sigma_{\mathrm{b}} \zeta^{2}-\sigma_{\mathrm{c}}\right)}$,

$\mathcal{P}_{\mathrm{s}}=\frac{-\sigma}{\zeta \sqrt{1-\zeta^{2}}+\sigma_{\mathrm{b}} \zeta^{2}-\sigma_{\mathrm{c}}}$.

Similarly, the shock ripple asymptotically oscillates according to

$\xi_{\mathrm{s}}(\tau \gg 1)= \begin{cases}\mathcal{J}_{\mathrm{lr}} \sin \left(\omega_{\mathrm{s}} \tau\right)+\mathcal{J}_{\mathrm{li}} \cos \left(\omega_{\mathrm{s}} \tau\right), & \zeta \leq 1, \\ \mathcal{J}_{\mathrm{s}} \sin \left(\omega_{\mathrm{s}} \tau\right), & \zeta \geq 1,\end{cases}$

with the corresponding coefficients being obtained through the shock-pressure variations as

$\mathcal{J}_{\mathrm{lr}}=\frac{\sigma_{\mathrm{a}}}{\omega_{\mathrm{s}}} \mathcal{P}_{\mathrm{lr}}+\frac{1}{\omega_{\mathrm{s}}}, \quad \mathcal{J}_{\mathrm{li}}=-\frac{\sigma_{\mathrm{a}}}{\omega_{\mathrm{s}}} \mathcal{P}_{\mathrm{li}}$

for the long-wavelength regime, and

$\mathcal{J}_{\mathrm{s}}=\frac{\sigma_{\mathrm{a}}}{\omega_{\mathrm{s}}} \mathcal{P}_{\mathrm{s}}+\frac{1}{\omega_{\mathrm{s}}}$

for the short-wavelength regime.

Only for $\zeta \geq 1$ the pressure perturbations escape from the shock in a stable manner. The velocity-acoustic perturbations are given by the long-time irrotational contribution, yielding

$\bar{u}_{\mathrm{a}}(x, \tau)=\mathcal{U}^{\mathrm{a}} \cos \left(\omega_{\mathrm{a}} \tau-k_{\mathrm{a}} x\right)$

and

$\bar{v}_{\mathrm{a}}(x, \tau)=\mathcal{V}^{\mathrm{a}} \sin \left(\omega_{\mathrm{a}} \tau-k_{\mathrm{a}} x\right)$,

with the

$\mathcal{U}^{\mathrm{a}}=\frac{k_{\mathrm{a}}}{\omega_{\mathrm{a}}} \mathcal{P}_{\mathrm{s}}, \quad \mathcal{V}^{\mathrm{a}}=\frac{1}{\omega_{\mathrm{a}}} \mathcal{P}_{\mathrm{s}}$,

being the associated amplitudes.

The asymptotic rotational contribution of the velocity field is

$\bar{u}_{\mathrm{r}}(x \gg 1)= \begin{cases}\mathcal{U}_{\mathrm{lr}}^{\mathrm{r}} \cos \left(\frac{\omega_{\mathrm{s}}}{M_{2}} x\right)+\mathcal{U}_{\mathrm{li}}^{\mathrm{r}} \sin \left(\frac{\omega_{\mathrm{s}}}{M_{2}} x\right), & \zeta \leq 1, \\ \mathcal{U}_{\mathrm{s}}^{\mathrm{r}} \cos \left(\frac{\omega_{\mathrm{s}}}{M_{2}} x\right), & \zeta \geq 1\end{cases}$

and

$\bar{v}_{\mathrm{r}}(x \gg 1)= \begin{cases}\mathcal{V}_{\mathrm{lr}}^{\mathrm{r}} \sin \left(\frac{\omega_{\mathrm{s}}}{M_{2}} x\right)+\mathcal{V}_{\mathrm{li}}^{\mathrm{r}} \cos \left(\frac{\omega_{\mathrm{s}}}{M_{2}} x\right), & \zeta \leq 1, \\ \mathcal{V}_{\mathrm{s}}^{\mathrm{r}} \sin \left(\frac{\omega_{\mathrm{s}}}{M_{2}} x\right), & \zeta \geq 1,\end{cases}$

with the amplitudes $\mathcal{U}^{\mathrm{r}}$ and $\mathcal{V}^{\mathrm{r}}$ being

$\mathcal{U}_{\mathrm{lr}}^{\mathrm{r}}=\frac{\Omega_{2} \mathcal{P}_{\mathrm{lr}}+\Omega_{1}}{1+\frac{1-M_{2}^{2}}{M_{2}^{2}} \zeta^{2}}, \quad \mathcal{U}_{\mathrm{li}}^{\mathrm{r}}=\frac{\Omega_{2} \mathcal{P}_{\mathrm{li}}}{1+\frac{1-M_{2}^{2}}{M_{2}^{2}} \zeta^{2}}$,

$\mathcal{U}_{\mathrm{s}}^{\mathrm{r}}=\frac{\Omega_{2} \mathcal{P}_{\mathrm{s}}+\Omega_{1}}{1+\frac{1-M_{2}^{2}}{M_{2}^{2}} \zeta^{2}}$

for the streamwise component, and

$\mathcal{V}_{\mathrm{lr}}^{\mathrm{r}}=\frac{\sqrt{1-M_{2}^{2}}}{M_{2}} \zeta \mathcal{U}_{\mathrm{lr}}^{\mathrm{r}}, \quad \mathcal{V}_{\mathrm{li}}^{\mathrm{r}}=-\frac{\sqrt{1-M_{2}^{2}}}{M_{2}} \zeta \mathcal{U}_{\mathrm{li}}^{\mathrm{r}}$,

$\mathcal{V}_{\mathrm{s}}^{\mathrm{r}}=\frac{\sqrt{1-M_{2}^{2}}}{M_{2}} \zeta \mathcal{U}_{\mathrm{s}}^{\mathrm{r}}$

for the crosswise component.
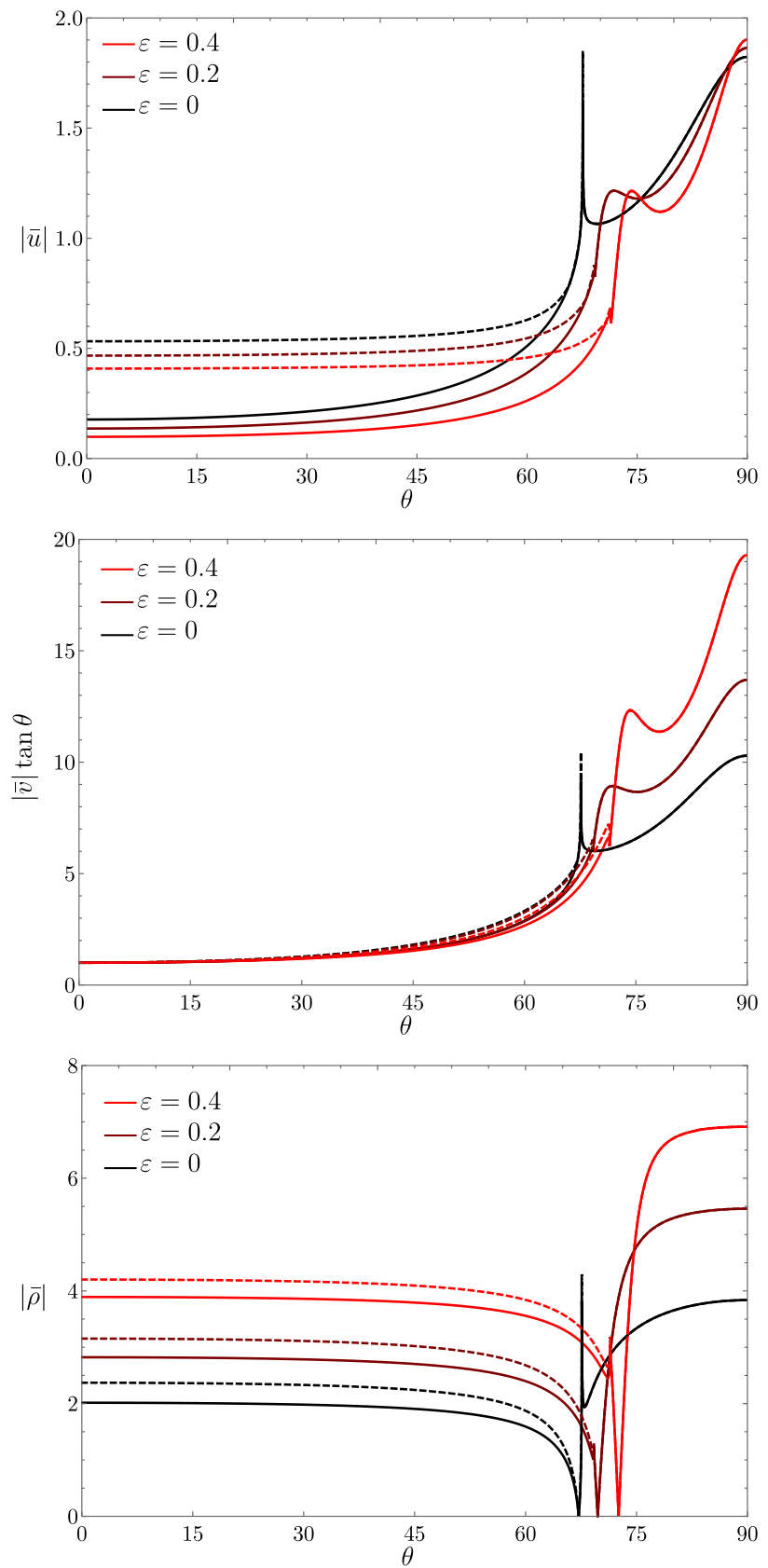

Figure A1. Velocity and density perturbation amplitudes for shock strength $M_{1}=5$ and for dissociation energy parameters $\varepsilon=0,0.2$, and 0.4 . Solid lines show the rotational-entropic contributions, while dashed lines include the effect of acoustic perturbations.

In the absence of pre-shock density perturbations, the amplitude of the density variations downstream is proportional to the shock pressure changes through equation (33), for the entropic contribution. The acoustic part coincides with the pressure field with the adimensionalization chosen. The total amplitude is then provided by the factor $\mathcal{D} \mathcal{P}$, with $\mathcal{P}$ given in equations (A15)-(A17).

The asymptotic value of the non-dimensional velocity and density amplitudes, as a function of the incident shear angle $\theta$, are shown in Fig. A1 for $\gamma=4 / 3, M_{1}=5$, and $\varepsilon=0,0.2$, and 0.4. Solid lines show the rotational-entropic contribution, and dashed lines include the acoustic contribution. Computations show that each of the amplitudes develops a pronounced finite-amplitude peak at the critical 
angle $\theta_{\text {cr }}$ corresponding to $\zeta=1$. The acoustic contribution is only important for low-incident angles (high-frequency disturbances) in the longitudinal component. The effect of dissociation energy in the peak is to shift the position to higher incident angles, and to make it less pronounced. The former, also found in Abdikamalov et al. (2016), is the result of the change induced by endothermic process in the background properties. The trend obviously reverses when studying exothermic reactive waves as detonations (Huete et al. 2017). The latter effect is however result of the perturbation of the energy employed in dissociating nuclei.

\section{APPENDIX B: NUCLEAR DISSOCIATION PARAMETER}

The nuclear dissociation at the shock can be parametrized in terms of the local free-fall velocity (Fernández \& Thompson 2009a,b):

$\Delta e_{\mathrm{dis}}=\frac{1}{2} \varepsilon v_{\mathrm{FF}}^{2}$,

where $\varepsilon$ is a dimensionless dissociation parameter. This parameter is related to the change of nuclear mass fractions in the following way. Consider nuclei with an atomic mass number $A_{i}$ in a fluid element of mass $M$ that contains multiple types of nuclei. Hereafter, index $i$ is used to refer to these nuclei. Suppose that these nuclei have a mass fraction of $X_{i}$ in the fluid element, meaning that the total mass of these nuclei in the fluid element is

$M_{i}=X_{i} M$.

The total number $N$ of these nuclei in the fluid element is then

$N=\frac{M_{i}}{A_{i} m_{i}}$,

where $m_{u}$ is the atomic mass unit. Suppose $e_{i}$ is the binding energy per nucleon for these nuclei. Thus, the binding energy per nucleus is $A e_{i}$. The total binding energy in the fluid element is simply $N A e_{i}$, from which one can easily obtain the binding energy per mass:

$E_{i}=\frac{N A e_{i}}{M}=\frac{M_{i}}{A_{i} m_{u}} \frac{A e_{i}}{M}=\frac{M_{i}}{M} \frac{e_{i}}{m_{u}}=X_{i} \frac{e_{i}}{m_{u}}$.

Thus, if the mass fraction of these nuclei changes by $\Delta X_{i}$ at shock crossing, the total binding energy per unit mass changes by

$\Delta E_{i}=\Delta X_{i} \frac{e_{i}}{m_{u}}$.

If $X_{i}<0$, i.e. the fraction of these nuclei decreases, then $\Delta E_{i}<0$, i.e. the total binding energy of the nuclei in the fluid decreases. This means that the fluid absorbs energy $-\Delta E_{i}$ for the dissociation per unit mass. The total dissociation energy at the shock is equal to the sum of $\Delta E_{i}$ given by equation (B5) for all the nuclei that exists in the fluid element:

$\Delta e_{\mathrm{dis}}=\sum_{i} \Delta E_{i}$

Thus, using equation (B1), the nuclear dissociation parameter can be calculated as

$\varepsilon=\frac{2 \Delta e_{\mathrm{dis}}}{m_{u} v_{\mathrm{FF}}^{2}}$.

In this work, the mass fractions are measured within 2-3 grid points above and below the shock. Extensive tests with different numbers of grid points have been performed in order to establish the validity of this approach.

This paper has been typeset from a $\mathrm{T}_{\mathrm{E}} \mathrm{X} / \mathrm{L} \mathrm{T} \mathrm{E} \mathrm{X}$ file prepared by the author. 